\title{
A cell-based smoothed discrete shear gap method (CS-DSG3) using triangular elements for static and free vibration analyses of shell structures
}

\author{
T. Nguyen-Thoi ${ }^{\mathrm{a}, \mathrm{b}, *}$, P. Phung-Van ${ }^{\mathrm{b}}, \mathrm{C}$. Thai-Hoang ${ }^{\mathrm{b}}$, H. Nguyen-Xuan ${ }^{\mathrm{a}, \mathrm{b}}$ \\ a Department of Mechanics, Faculty of Mathematics \& Computer Science, University of Science Vietnam National University HCMC, 227 Nguyen Van Cu, \\ District 5, Hochiminh City, Vietnam \\ ${ }^{\mathrm{b}}$ Division of Computational Mechanics, Ton Duc Thang University, Nguyen Huu Tho Street, Tan Phong Ward, District 7, Hochiminh City, Vietnam
}

\section{A R T I C L E I N F O}

\section{Article history:}

Received 20 January 2012

Received in revised form

11 January 2013

Accepted 24 April 2013

Available online 20 May 2013

\section{Keywords:}

Reissner-Mindlin shell

Shear locking

Cell-based smoothed discrete shear gap

technique (CS-DSG3)

Smoothed finite element method

Strain smoothing technique

\begin{abstract}
A B S T R A C T
A cell-based smoothed discrete shear gap method (CS-DSG3) for static and free vibration analyses of Reissner-Mindlin shells is formulated by combining the cell-based strain smoothing technique with the discrete shear gap method (DSG3) using three-node triangular elements. In the CS-DSG3, each triangular element will be divided into three sub-triangles, and in each sub-triangle, the stabilized DSG3 is used to compute the strains and to avoid the transverse shear locking. Then the strain smoothing technique on whole of the triangular element is used to smooth the strains on these three sub-triangles. The CS-DSG3 hence not only overcomes the drawback of the DSG3 but also improves the accuracy as well as the stability of the DSG3. The numerical examples demonstrated that the CS-DSG3 is free of shear locking and achieves the high accuracy compared to other existing shell elements.
\end{abstract}

(c) 2013 Elsevier Ltd. All rights reserved.

\section{Introduction}

The numerical analysis of shell structures in large-scale industrial problems has always been a challenge and receives continuously strong interest [1-3]. In the past three decades, the finite element method (FEM) has been used as a powerful numerical tool to simulate behaviors of shell structures [4]. Compared with four-node quadrilateral shell element [5-8], three-node triangular shell element $[9,10]$ is particularly attractive due to its simplicity, automatic meshing and re-meshing in adaptive analysis. However, the derivation of simple and effective three-node triangular shell elements for analysis of general shell structures with complex loading and boundary conditions is still a challenging research topic. This paper hence focuses on developing such a simple and effective three-node triangular shell element.

Based on the theories of formulation [1], shell elements can be classified into three main groups: (1) degenerated shell elements derived from the three-dimensional (3D) solid theory; (2) curved shell elements based on general shell theory; and (3) flat shell

\footnotetext{
* Corresponding author at: Faculty of Mathematics \& Computer Science, University of Science HCM, 227 Nguyen Van Cu, Dist. 5, Hochiminh City, Vietnam. Tel.: +84942340411.

E-mail addresses: ngttrung@hcmus.edu.vn, thoitrung76@gmail.com (T. Nguyen-Thoi).
}

elements formulated by combining a plane elastic membrane element and a plate bending element. Among these three groups, the flat shell elements are rather popular due to simple formulation and low computational cost, and hence the theory of flat shell elements will be chosen to develop the triangular shell element in this paper.

Shell elements can also be classified according to the thin shell elements and thick shell elements [1,2]. Thin shell elements are based on the Kirchhoff-Love theory in which transverse shear deformations are neglected, while thick shell elements are based on the Reissner-Mindlin theory which includes transverse shear deformations. The thin shell elements are limited only for thin shells and require C1 continuity for the deflection field which makes the formulation become more difficult. While the thick shell elements, or Reissner-Mindlin shell elements, can be used for both thin and thick shells and only require $\mathrm{CO}$ continuity for the deflection and rotation fields which make the formulation become easier. However, three-node triangular Reissner-Mindlin shell elements often suffer from two major drawbacks; (1) the so called "shear locking" phenomena which pollutes the numerical results in the thin limit; and (2) the overly stiff behavior which leads to poor accuracy and low convergence of numerical solutions.

Shear locking is caused by parasitic shear deformation energy which leads to an artificial additional stiffness as the shells becomes progressively thinner. This drawback can be overcome by using some different methods such as reduced integration [11] or assumed 
natural strains (ANS) [12]. In the methods using reduced integration, different integration rules are used for the bending strain energy and shear strain energy. While in the methods using the ANS, the shear strains are not computed directly from the derivatives of displacements over the element but from additional interpolation based on specific shape functions and the derivatives of displacements at only some discrete points. The shell elements using reduced integration, however, often give low accuracy and exhibit zero energy modes, and hence stabilized techniques [5] are necessary to eliminate these modes. While the elements using ANS often give more satisfied and stable results. As a result, various shell elements have been developed following this trend $[13,14]$.

Recently, Bletzinger et al. [15] proposed a three-node triangular shell element DSG3 based on the Discrete Shear Gap method (DSG) which can be classified as an ANS element. In the DSG3, the shear strain is linear interpolated from the shear gaps of displacement along the sides of the elements by using the standard element shape functions. The DSG3 shell element can satisfy explicitly the kinematic equation for the shear strains at discrete points and effectively eliminates the parasitic shear strains. However, the element stiffness matrix in the DSG3 still depends on the sequence of node numbers, and hence the solution of DSG3 is influenced when the sequence of node numbers changes, especially for the coarse and distorted meshes.

The overly stiff behavior is usually observed in many ReissnerMindlin shell elements based on the compatible displacement-based FEM models. The overly stiff behavior is even more severe when three-node triangular shell elements are used. In order to reduce the overly stiffness of the displacement-based FEM models, Liu et al. [16-18] proposed a cell-based smoothed finite element method (CS-FEM) which is a combination of the standard FEM and a strain smoothing technique [19] used in meshfree methods. In the CS-FEM, the domain discretization is still based on elements as in the FEM; however the stiffness matrices are calculated based over smoothing domains located inside the elements. The CS-FEM, however so far, has been developed only for the 4-node quadrilateral elements [20-25] and the improvement of accuracy of solutions compared to those of the existing quadrilateral elements is still marginal.

This paper hence extends the CS-FEM for triangular elements and for significant improvement of solutions of shell analysis. The cell-based strain smoothing technique in the CS-FEM is combined with the DSG3 [15] using three-node triangular elements to give a so-called the cell-based smoothed discrete shear gap method (CS-DSG3) for static and free vibration analyses of ReissnerMindlin shells. In the CS-DSG3, each triangular element will be divided into three sub-triangles, and in each sub-triangle the stabilized DSG3 [26] is used to compute the strains and to avoid the transverse shear locking. Then the strain smoothing technique on whole the triangular element is used to smooth the strains on these three sub-triangles. The accuracy and reliability of the proposed method is verified by comparing its numerical solutions with those of others available numerical results.

\section{Weakform and general FEM formulation of the Reissner-Mindlin shell}

The shell element is subjected to both membrane forces and bending forces and hence the development of flat shell elements should be a combination of a membrane element and a plate bending element. In the following sections, we brief on the weakform and general FEM formulation of Reissner-Mindlin shell elements.

\subsection{Weak form of the Reissner-Mindlin shell}

Consider a shell subjected to both membrane forces and bending forces. The middle (neutral) surface of plate is chosen as the reference plane that occupies a domain $\Omega \in \mathbb{R}^{3}$ as shown in Fig. 1. Let $u, v, w$ be the displacements of the middle plane in the

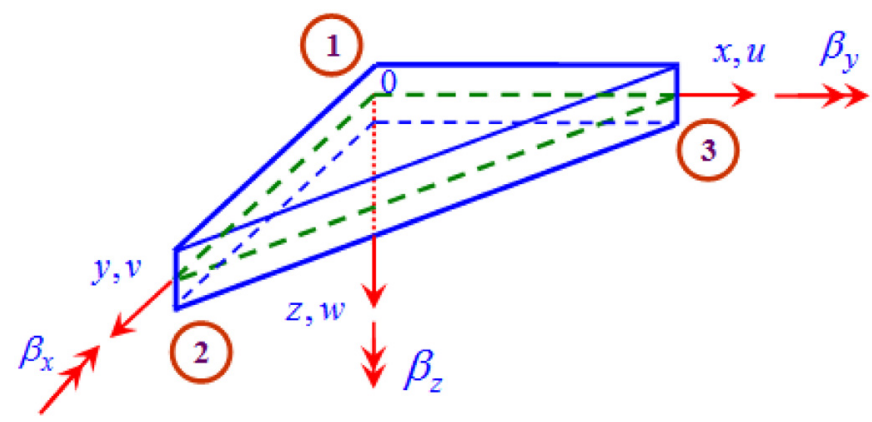

Fig. 2. Three-node triangular element.

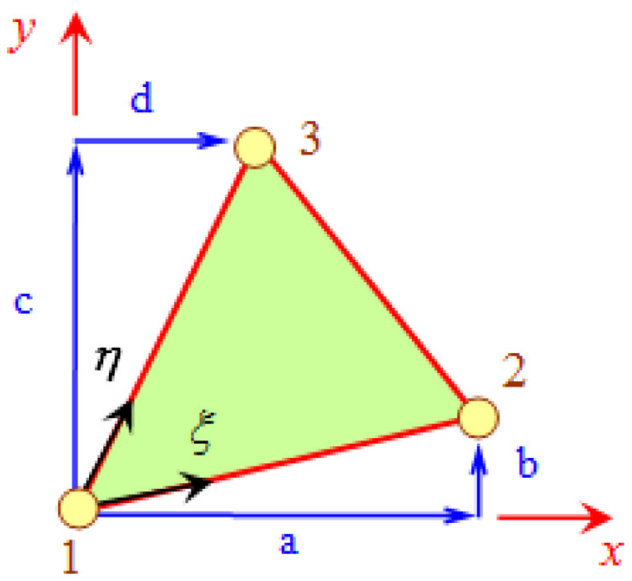

Fig. 3. Three-node triangular element and local coordinates in the DSG3.
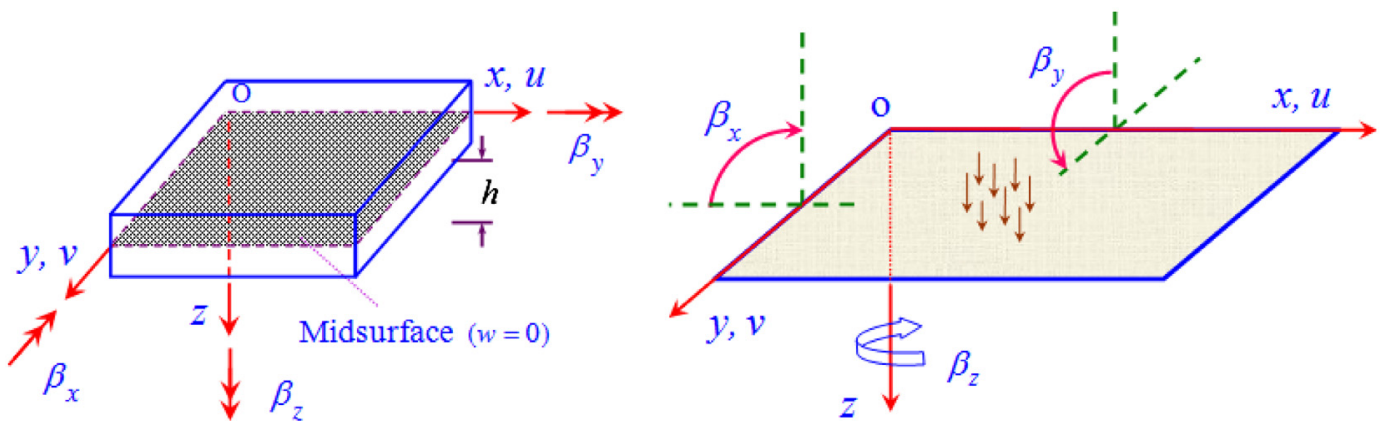

Fig. 1. Reissner-Mindlin flat plate and positive directions of the displacement $w$ and three rotations $\beta_{x}, \beta_{y}$ and $\beta_{z}$. 
$x, y, z$ directions, and $\beta_{x}, \beta_{y}, \beta_{z}$ are the rotations of the middle plane around $y$-axis, $x$-axis, and $z$-axis respectively, with the positive directions defined as shown in Fig. 1.

The unknown vector of six independent field variables at any point in the problem domain of the Reissner-Mindlin shells can be written as

$\mathbf{u}=\left[\begin{array}{llllll}u & v & w & \beta_{x} & \beta_{y} & \beta_{z}\end{array}\right]^{T}$

The membrane strain $\varepsilon^{m}$, the curvature of the shell $\kappa$ and the shear strains $\gamma$ are defined, respectively, as

$\left(\varepsilon^{m}\right)^{T}=\left[\begin{array}{lll}\frac{\partial u}{\partial x} & \frac{\partial v}{\partial y} & \frac{\partial u}{\partial y}+\frac{\partial v}{\partial x}\end{array}\right] ; \quad \boldsymbol{\kappa}^{T}=\left[\begin{array}{lll}\frac{\partial \beta_{x}}{\partial x} & \frac{\partial \beta_{y}}{\partial y} & \frac{\partial \beta_{x}}{\partial y}+\frac{\partial \beta_{y}}{\partial x}\end{array}\right] ; \quad \boldsymbol{\gamma}=\left[\begin{array}{l}\frac{\partial w}{\partial x}+\beta_{x} \\ \frac{\partial w}{\partial y}+\beta_{y}\end{array}\right]$

The standard Galerkin weakform of the static equilibrium equations for the Reissner-Mindlin shell can now be written as [1-3]

$\int_{\Omega}\left(\delta \varepsilon^{m}\right)^{T} \mathbf{D}^{m} \varepsilon^{m} \mathrm{~d} \Omega+\int_{\Omega} \delta \boldsymbol{\kappa}^{T} \mathbf{D}^{b} \boldsymbol{\kappa} \mathrm{d} \Omega+\int_{\Omega} \delta \boldsymbol{\gamma}^{T} \mathbf{D}^{S} \boldsymbol{\gamma} \mathrm{d} \Omega=\int_{\Omega} \delta \mathbf{u}^{T} \mathbf{b} \mathrm{d} \Omega$

where $\mathbf{b}=\left[\begin{array}{llllll}0 & 0 & p(x, y, z) & 0 & 0 & 0\end{array}\right]^{T}$ is the distributed load applied on the shell. The matrices $\mathbf{D}^{m}, \mathbf{D}^{b}$ and $\mathbf{D}^{s}$ are, respectively, the material matrices related to the membrane deformation, bending deformation and shear deformation, and are given by

$\mathbf{D}^{m}=\frac{E t}{\left(1-v^{2}\right)}\left[\begin{array}{ccc}1 & v & 0 \\ v & 1 & 0 \\ 0 & 0 & (1-v) / 2\end{array}\right]$

$\mathbf{D}^{b}=\frac{E t^{3}}{12\left(1-v^{2}\right)}\left[\begin{array}{ccc}1 & v & 0 \\ v & 1 & 0 \\ 0 & 0 & (1-v) / 2\end{array}\right] ; \quad \mathbf{D}^{s}=k t\left[\begin{array}{cc}\mu & 0 \\ 0 & \mu\end{array}\right]$

where $E$ is Young's modulus; $t$ is the thickness of plate; $v$ is the poisson constant; $\mu$ is the shear modulus and $k=5 / 6$ is the shear correction factor.

For the free vibration analysis of Reissner-Mindlin shells, the standard Galerkin weakform can be derived from the dynamic form of energy principle [1-3]

$\int_{\Omega}\left(\delta \boldsymbol{\varepsilon}^{m}\right)^{T} \mathbf{D}^{m} \boldsymbol{\varepsilon}^{m} \mathrm{~d} \Omega+\int_{\Omega} \delta \boldsymbol{\kappa}^{T} \mathbf{D}^{b} \boldsymbol{\kappa} \mathrm{d} \Omega+\int_{\Omega} \delta \boldsymbol{\gamma}^{T} \mathbf{D}^{S} \boldsymbol{\gamma} \mathrm{d} \Omega+\int_{\Omega} \delta \mathbf{u}^{T} \mathbf{m} \ddot{\mathbf{u}} \mathrm{d} \Omega=0$

where $\mathbf{m}$ is the matrix containing the mass density of the material $\rho$ and thickness $t$ as

$\mathbf{m}=\operatorname{diag}\left[\rho t, \rho t, \rho t, \rho t^{3} / 12, \rho t^{3} / 12,0\right]$

\subsection{General FEM formulation of Reissner-Mindlin flat shell elements}

Now, discretize the bounded domain $\Omega$ into $N_{e}$ finite elements such that $\Omega=\mathrm{U}_{e=1}^{N_{e}} \Omega_{e}$ and $\Omega_{i} \cap \Omega_{j}=\varnothing, i \neq j$, then the finite element solution $\mathbf{u}^{h}=\left[\begin{array}{llllll}u & v & w & \beta_{x} & \beta_{y} & \beta_{z}\end{array}\right]^{T}$ of a displacement model for the Reissner-Mindlin shell is expressed as

$\mathbf{u}^{h}=\sum_{I=1}^{N_{n}} \underbrace{N_{I}(\mathbf{x}) \mathbf{I}_{6}}_{\mathbf{N}_{I}} \mathbf{d}_{I}=\sum_{I=1}^{N_{n}} \mathbf{N}_{I} \mathbf{d}_{I}$

where $\mathbf{I}_{6}$ is the unit matrix of 6th rank; $N_{n}$ is the total number of nodes of problem domain discretized; $N_{I}(\mathbf{x})$ is the shape function at the Ith node; $\mathbf{d}_{I}=\left[\begin{array}{llllll}u_{I} & v_{I} & w_{I} & \beta_{x I} & \beta_{y I} & \beta_{z I}\end{array}\right]^{T}$ is the displacement vector of the nodal degrees of freedom of $\mathbf{u}^{h}$ associated to the Ith node.
The membrane, bending and shear strains can be then expressed in the matrix forms as

$\varepsilon^{m}=\sum_{I} \mathbf{R}_{I} \mathbf{d}_{I}, \quad \boldsymbol{\kappa}=\sum_{I} \mathbf{R}_{I} \mathbf{d}_{I}, \quad \boldsymbol{\gamma}^{s}=\sum_{I} \mathbf{S}_{I} \mathbf{d}_{I}$

where

$\mathbf{R}_{I}=\left[\begin{array}{cccccc}N_{I, X} & 0 & 0 & 0 & 0 & 0 \\ 0 & N_{I, y} & 0 & 0 & 0 & 0 \\ N_{I, y} & N_{I, X} & 0 & 0 & 0 & 0\end{array}\right]$

$\mathbf{B}_{I}=\left[\begin{array}{cccccc}0 & 0 & 0 & N_{I, X} & 0 & 0 \\ 0 & 0 & 0 & 0 & N_{I, y} & 0 \\ 0 & 0 & 0 & N_{I, y} & N_{I, X} & 0\end{array}\right]$

$\mathbf{S}_{I}=\left[\begin{array}{cccccc}0 & 0 & N_{I, x} & N_{I} & 0 & 0 \\ 0 & 0 & N_{I, y} & 0 & N_{I} & 0\end{array}\right]$

The discretized system of equations of the Reissner-Mindlin shell using the FEM for static analysis then can be expressed as

$\mathbf{K d}=\mathbf{F}$

where $\mathbf{K}$ is the global stiffness matrix given by

$\mathbf{K}=\int_{\Omega} \mathbf{R}^{T} \mathbf{D}^{m} \mathbf{R} \mathrm{d} \Omega+\int_{\Omega} \mathbf{B}^{T} \mathbf{D}^{b} \mathbf{B} \mathrm{d} \Omega+\int_{\Omega} \mathbf{S}^{T} \mathbf{D}^{s} \mathbf{S} \mathrm{d} \Omega$

and $\mathbf{F}$ is the load vector defined as

$\mathbf{F}=\int_{\Omega} p \mathbf{N} \mathrm{d} \Omega+\mathbf{f}^{b}$

in which $\mathbf{f}^{b}$ is the remaining part of $\mathbf{F}$ subjected to prescribed boundary loads.

For free vibration analysis, we have

$\left(\mathbf{K}-\omega^{2} \mathbf{M}\right) \mathrm{d}=\mathbf{0}$

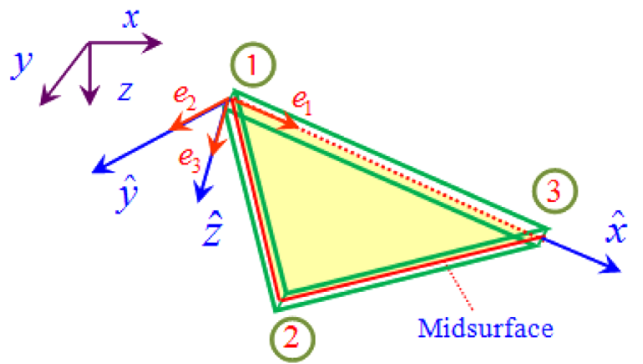

Fig. 4. Coordinate transformation in the triangular flat shell elements.

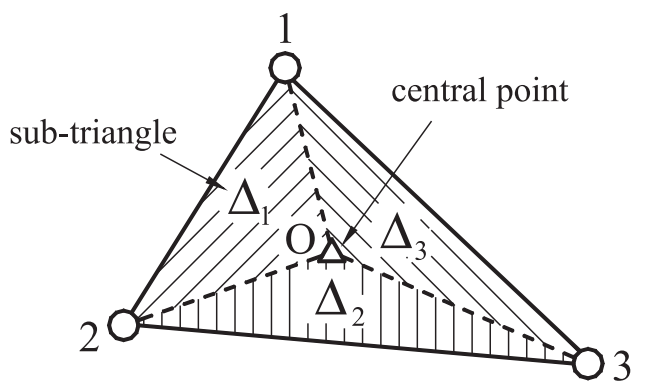

Fig. 5. Three sub-triangles $\left(\Delta_{1}, \Delta_{2}\right.$ and $\left.\Delta_{3}\right)$ created from the triangle 1-2-3 in the CS-DSG3 by connecting the central point $O$ with three field nodes 1,2 and 3 . 
where $\omega$ is the natural frequency and $\mathbf{M}$ is the global mass matrix defined by

$\mathbf{M}=\int_{\Omega} \mathbf{N}^{T} \mathbf{m} \mathbf{N}^{T} \mathrm{~d} \Omega$

\section{Formulation of the three-node triangular flat shell element CS-DSG3}

In this section, the three-node triangular flat shell element CS-DSG3 is formulated by combining the cell-based strain smoothing technique $[17,18]$ with the stabilized discrete shear gap method (DSG3) using three-node triangular elements [15].

\subsection{Brief on the DSG3 formulation}

The formulation of the stabilized DSG3 [15] is based on the concept "shear gap" of displacement along the sides of the elements. In the DSG3, the shear strain is linear interpolated from the shear gaps of displacement by using the standard element shape functions. As a result, the operator matrix $\mathbf{S}$ related to shear part is modified, and its entries are constant and computed from the coordinates of nodes of elements. The DSG3 element is shearlocking-free and has several superior properties as presented in Ref [15]. In this paper, we just brief on the DSG3 formulation which is necessary for the formulation of the CS-DSG3.

Using a mesh of triangular elements, the approximation $\mathbf{u}_{e}^{h}=\left[\begin{array}{llllll}u_{e}^{h} & v_{e}^{h} & w_{e}^{h} & \beta_{e x}^{h} & \beta_{e y}^{h} & \beta_{e z}^{h}\end{array}\right]^{T}$ for a 3-node triangular shell

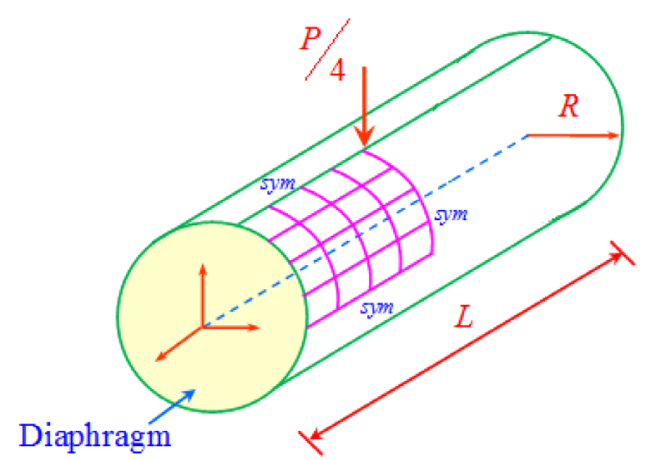

Fig. 6. Geometry of the pinched cylinder with diaphragms boundary conditions. element $\Omega_{e}$ shown in Fig. 2 can be written as

$\mathbf{u}_{e}^{h}=\sum_{I=1}^{3} \underbrace{N_{I}(\mathbf{x}) \mathbf{I}_{6}}_{\mathbf{N}_{l}} \mathbf{d}_{e I}=\sum_{I=1}^{3} \mathbf{N}_{I} \mathbf{d}_{e I}$

where $\mathbf{d}_{e I}=\left[\begin{array}{llllll}u_{I} & v_{I} & w_{I} & \beta_{x I} & \beta_{y I} & \beta_{z I}\end{array}\right]^{T}$ are the nodal degrees of freedom of $\mathbf{u}_{e}^{h}$ associated to the Ith node and $N_{I}(\mathbf{x})$ are linear shape functions in a natural coordinate defined by

$N_{1}=1-\xi-\eta, \quad N_{2}=\xi, \quad N_{3}=\eta$

The membrane strain and curvatures of the deflection in the element are then obtained by

$\boldsymbol{\varepsilon}^{m h}=\underbrace{\left[\begin{array}{lll}\mathbf{R}_{1} & \mathbf{R}_{2} & \mathbf{R}_{3}\end{array}\right]}_{\mathbf{R}} \mathbf{d}_{e}=\mathbf{R d}_{e}$

$\boldsymbol{\kappa}^{h}=\underbrace{\left[\begin{array}{lll}\mathbf{B}_{1} & \mathbf{B}_{2} & \mathbf{B}_{3}\end{array}\right]}_{\mathbf{B}} \mathbf{d}_{e}=\mathbf{B} \mathbf{d}_{e}$

where $\mathbf{d}_{e}=\left[\begin{array}{lll}\mathbf{d}_{e 1} & \mathbf{d}_{e 2} & \mathbf{d}_{e 3}\end{array}\right]^{T}$ is the nodal displacement vector of element; $\mathbf{R}$ and $\mathbf{B}$ contains the derivatives of the shape functions that are constants in which

$\mathbf{R}_{1}=\left[\begin{array}{cccccc}b-c & 0 & 0 & 0 & 0 & 0 \\ 0 & d-a & 0 & 0 & 0 & 0 \\ d-a & b-c & 0 & 0 & 0 & 0\end{array}\right] ; \quad \mathbf{R}_{2}=\left[\begin{array}{cccccc}c & 0 & 0 & 0 & 0 & 0 \\ 0 & -d & 0 & 0 & 0 & 0 \\ -d & c & 0 & 0 & 0 & 0\end{array}\right] ;$

$\mathbf{R}_{3}=\left[\begin{array}{cccccc}-b & 0 & 0 & 0 & 0 & 0 \\ 0 & a & 0 & 0 & 0 & 0 \\ a & -b & 0 & 0 & 0 & 0\end{array}\right]$

$\mathbf{B}_{1}=\left[\begin{array}{cccccc}0 & 0 & 0 & b-c & 0 & 0 \\ 0 & 0 & 0 & 0 & d-a & 0 \\ 0 & 0 & 0 & d-a & b-c & 0\end{array}\right] ; \quad \mathbf{B}_{2}=\left[\begin{array}{cccccc}0 & 0 & 0 & c & 0 & 0 \\ 0 & 0 & 0 & 0 & -d & 0 \\ 0 & 0 & 0 & -d & c & 0\end{array}\right] ;$

$\mathbf{B}_{3}=\left[\begin{array}{cccccc}0 & 0 & 0 & -b & 0 & 0 \\ 0 & 0 & 0 & 0 & a & 0 \\ 0 & 0 & 0 & a & -b & 0\end{array}\right]$

with $a=x_{2}-x_{1}, b=y_{2}-y_{1}, c=y_{3}-y_{1}, d=x_{3}-x_{1}$ as shown in Fig. 3, and $\mathbf{x}_{i}=\left[\begin{array}{ll}x_{i} & y_{i}\end{array}\right]^{T}, i=1,2,3$, are coordinates of three nodes in the local coordinate system, respectively and $A_{e}$ is the area of the triangular element.

As reported in literatures on Reissner-Mindlin elements, the shear locking often occurs when the thickness of shell becomes small, where the pure bending dominates the shell deformation.
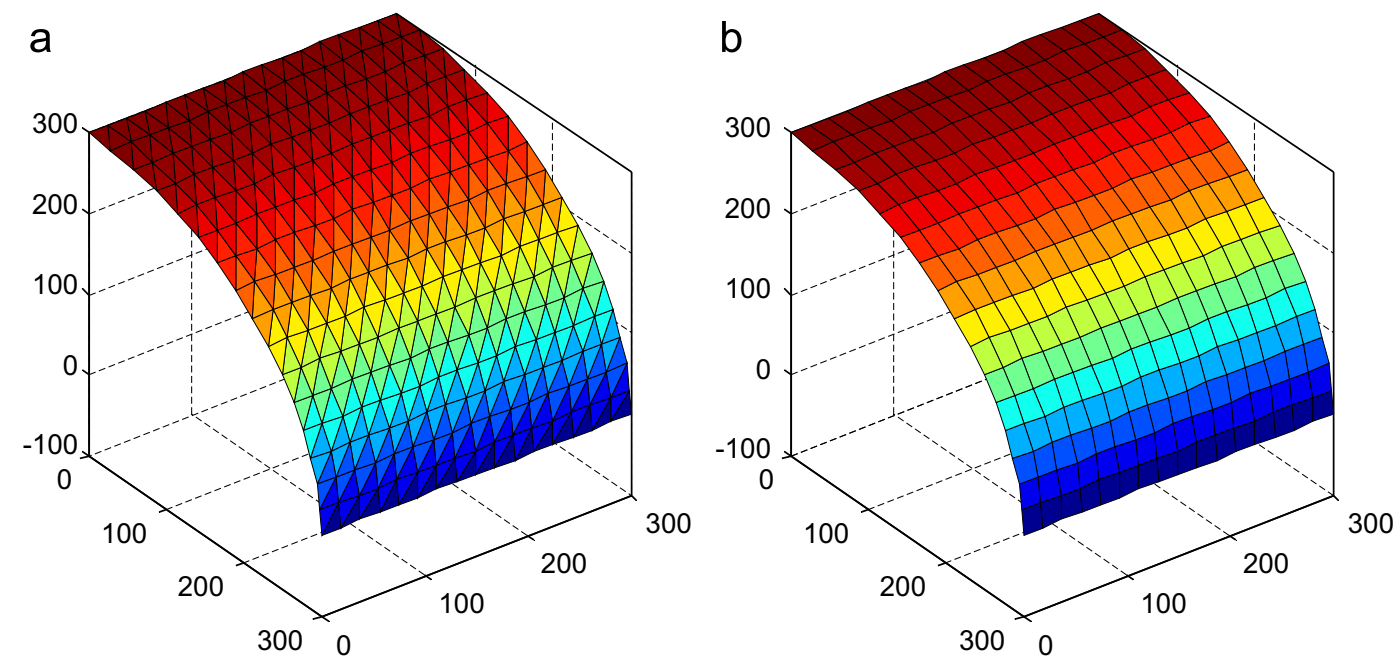

Fig. 7. Two discretizations of one eighth of the Pinched cylinder with diaphragms boundary conditions using (a) triangular elements and (b) quadrilateral elements. 
This is because the parasitic transverse shear strains are not eliminated under pure bending conditions. In order to overcome this conflict, Bletzinger et al. [15] proposed the discrete shear gap method (DSG3) to alter the shear strain field. The altered shear strains are in the form of

$\boldsymbol{\gamma}^{h}=\underbrace{\left[\begin{array}{lll}\mathbf{S}_{1} & \mathbf{S}_{2} & \mathbf{S}_{3}\end{array}\right]}_{\mathbf{S}} \mathbf{d}_{e}=\mathbf{S} \mathbf{d}_{e}$

where

$\mathbf{S}_{1}=\frac{1}{2 A_{e}}\left[\begin{array}{cccccc}0 & 0 & b-c & A_{e} & 0 & 0 \\ 0 & 0 & d-a & 0 & A_{e} & 0\end{array}\right]$

$\mathbf{S}_{2}=\frac{1}{2 A_{e}}\left[\begin{array}{cccccc}0 & 0 & c & a c / 2 & b c / 2 & 0 \\ 0 & 0 & -d & -a d / 2 & -b d / 2 & 0\end{array}\right]$

$\mathbf{S}_{3}=\frac{1}{2 A_{e}}\left[\begin{array}{cccccc}0 & 0 & -b & -b d / 2 & -b c / 2 & 0 \\ 0 & 0 & a & a d / 2 & a c / 2 & 0\end{array}\right]$

Substituting matrices R, B, and $\mathbf{S}$ in Eqs. (19), (20) and (23), respectively, into Eq. (13), the global stiffness matrix now becomes

$\mathbf{K}^{D S G 3}=\sum_{e=1}^{N_{e}} \mathbf{K}_{e}^{D S G 3}$

where $\mathbf{K}_{e}^{D S G 3}$ is the element stiffness matrix of the DSG3 element and is given by

$$
\begin{aligned}
\mathbf{K}_{e}^{D S G 3} & =\mathbf{T}^{T}\left(\int_{\Omega_{e}} \mathbf{R}^{T} \mathbf{D}^{m} \mathbf{R} \mathbf{d} \Omega+\int_{\Omega_{e}} \mathbf{B}^{T} \mathbf{D}^{b} \mathbf{B} \mathbf{d} \Omega+\int_{\Omega_{e}} \mathbf{S}^{T} \mathbf{D}^{s} \mathbf{S} \mathbf{d} \Omega\right) \mathbf{T} \\
& =\mathbf{T}^{T} \underbrace{\left(\mathbf{R}^{T} \mathbf{D}^{m} \mathbf{R} A_{e}+\mathbf{B}^{T} \mathbf{D}^{b} \mathbf{B} A_{e}+\mathbf{S}^{T} \mathbf{D}^{S} \mathbf{S} A_{e}\right)}_{\mathbf{k}_{e}} \mathbf{T} \\
& =\mathbf{T}^{T} \mathbf{k}_{e} \mathbf{T}
\end{aligned}
$$

in which $\mathbf{k}_{e}$ is the element stiffness matrix computed in the local coordinate system $\hat{x} \hat{y} \hat{z}$, and $\mathbf{T}$ is the transformation matrix of coordinates from the global coordinate system $x y z$ to the local coordinate system $\hat{x} \hat{y} \hat{z}$ as shown in Fig. 4.

It was suggested [26] that a stabilization term needs to be added to the original DSG3 element to further improve the accuracy of approximate solutions and to stabilize shear force oscillations. Such a modification is achieved by simply replacing $\mathbf{D}^{s}$ in Eq. (28) by $\hat{\mathbf{D}}^{s}$ as

$\hat{\mathbf{D}}^{s}=\frac{k t^{3}}{t^{2}+\alpha h_{e}^{2}}\left[\begin{array}{ll}1 & 0 \\ 0 & 1\end{array}\right]$

in which $h_{e}$ is the longest length of the edges of the element and $\alpha$ is a positive constant [27].

From Eqs. (19), (20), (23) and (28), it is clear that the values of element stiffness matrix at the drilling degree of freedom $\beta_{z}$ equal zero which can cause the singularity in the global stiffness matrix when all the elements meeting at a node are coplanar. To deal with this issue, the null values of the stiffness corresponding to the drilling degree of freedom are then replaced by approximate values. This approximate value is taken to be equal to $10^{-3}$ times the maximum diagonal value in the element stiffness matrix [25].

Also from Eqs. (19), (20), (23) and (28), it is seen that the element stiffness matrix in the DSG3 depends on the sequence of node numbers of elements, and hence the solution of DSG3 is influenced when the sequence of node numbers of elements changes, especially for the coarse and distorted meshes. The CS-DSG3 is hence proposed to overcome this drawback and also to improve the accuracy as well as the stability of the DSG3.

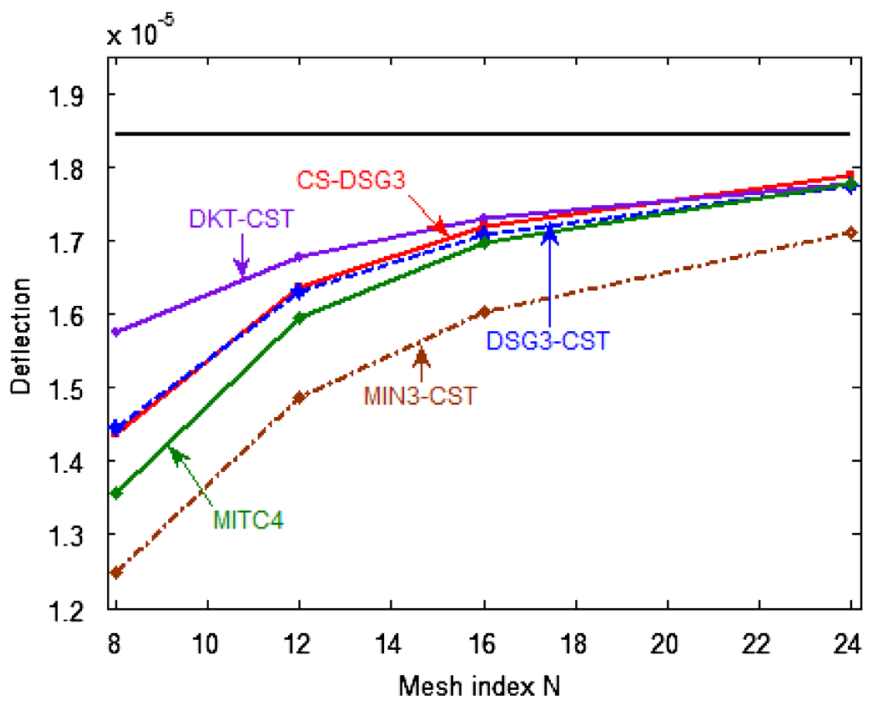

Fig. 8. Convergence of the central deflection at point A of the Pinched cylinder obtained using structured meshes and different methods.

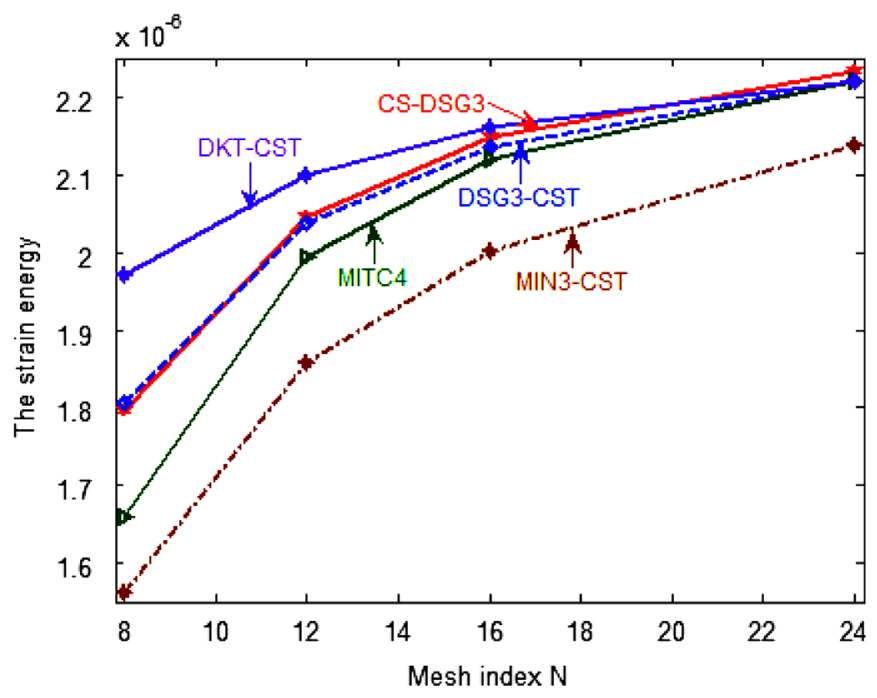

Fig. 9. Convergence of the strain energy of the Pinched cylinder obtained using structured meshes and different methods.

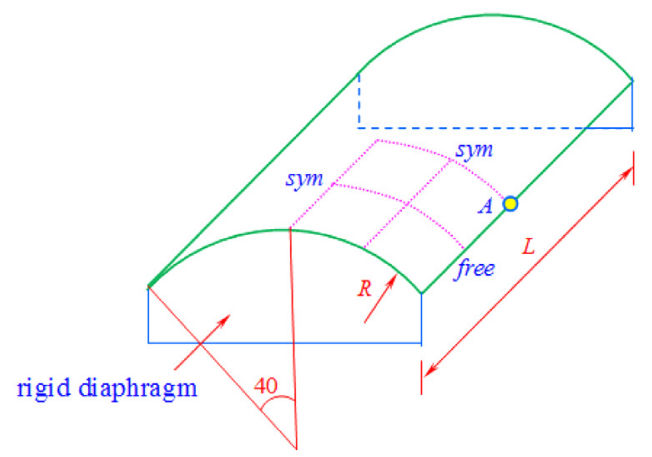

Fig. 10. Geometry of the Scordelis-Lo roof in which two curved edges are supported by rigid diaphragms $(u=w=0)$, and the other two edges are free. 

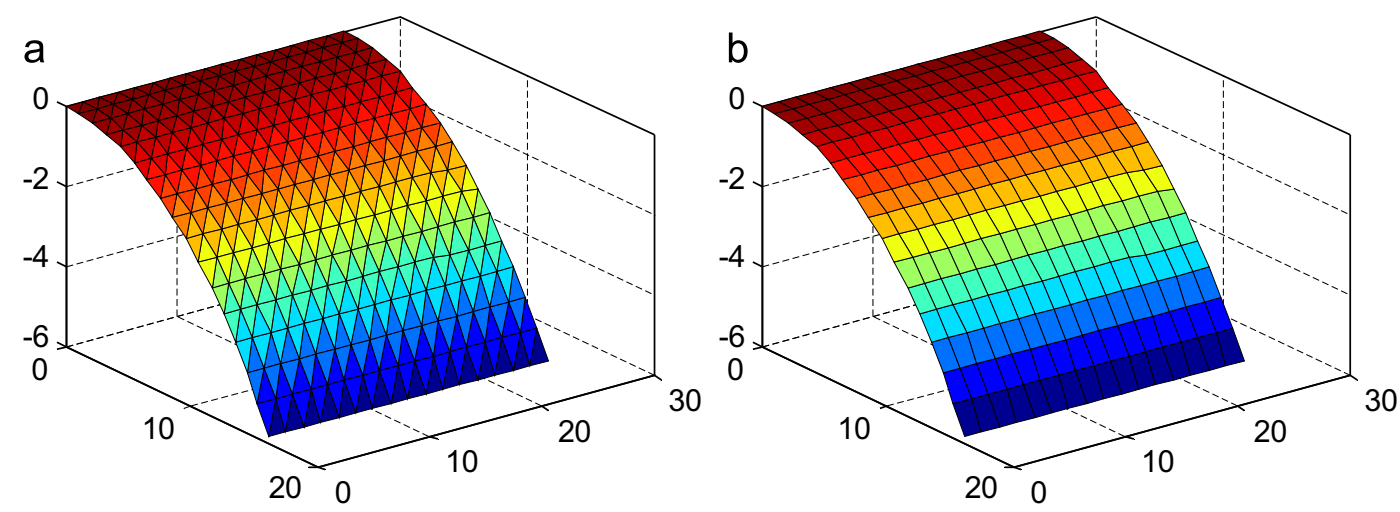

Fig. 11. Two discretizations of a quarter of the Scordelis-Lo roof using (a) triangular elements and (b) quadrilateral elements.

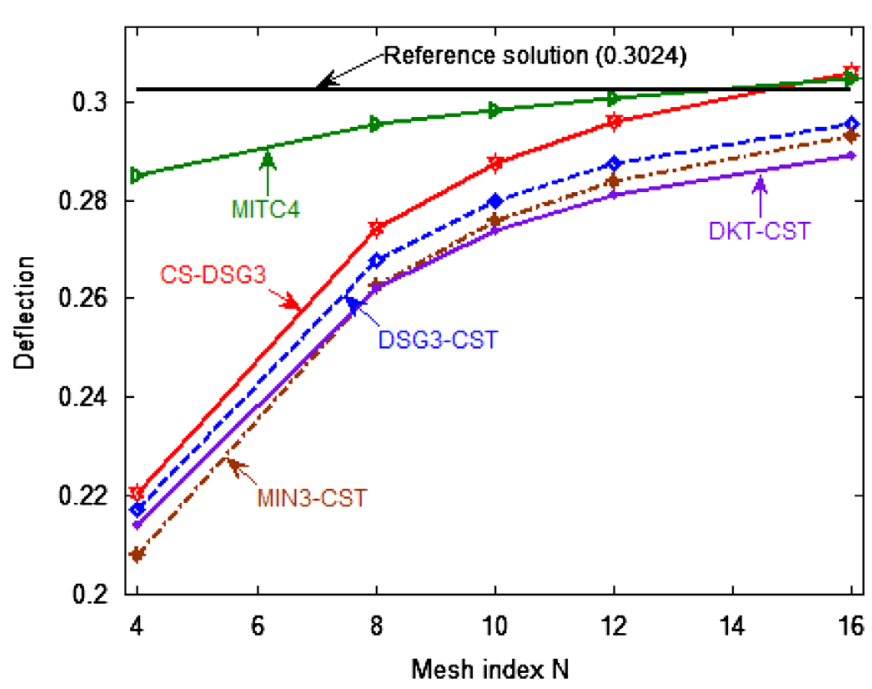

Fig. 12. Convergence of the mid-side vertical displacement at point $A$ of the Scordelis-Lo roof obtained using structured meshes and different methods.

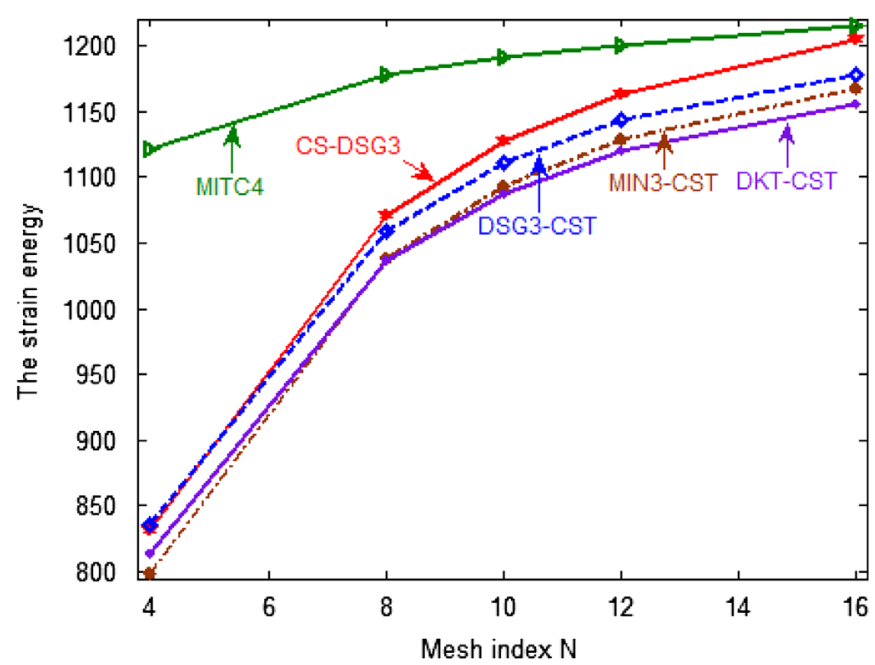

Fig. 13. Convergence of the strain energy of the Scordelis-Lo roof obtained using structured meshes and different methods.

\subsection{Formulation of the flat shell element CS-DSG3}

In the CS-DSG3, each triangular element is divided into three sub-triangles by connecting the central point of the element to three field nodes, and the displacement vector at the central point is assumed to be the simple average of three displacement vectors

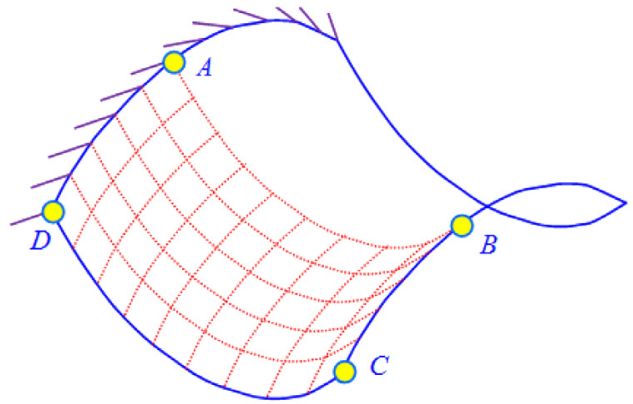

Fig. 14. Geometry of the partly clamped hyperbolic paraboloid shell structure in which one side is clamped and three other edges are free.

of three field nodes. In each sub-triangles, the stabilized DSG3 is used to compute the strains and also to avoid the transverse shear locking. Then the strain smoothing technique on whole of the triangular element is used to smooth the strains on these three sub-triangles. The formulation of CS-DSG3 is presented in detail as follows:

Consider a typical triangular element $\Omega_{e}$ as shown in Fig. 5. We first divide the element into 3 sub-triangles $\Delta_{1}, \Delta_{2}$ and $\Delta_{3}$ such as $\Omega_{e}=\mathrm{U}_{i=1}^{3} \Delta_{i}$ and $\Delta_{i} \cap \Delta_{j}=\varnothing, i \neq j$, by simply connecting the central point $O$ of the triangle with 3 field nodes as shown in Fig. 5.

In the CS-DSG3, we assume that the displacement vector $\mathbf{d}_{e O}$ at the central point $O$ is the simple average of three displacement vectors $\mathbf{d}_{e 1}, \mathbf{d}_{e 2}$ and $\mathbf{d}_{e 3}$ of three field nodes

$\mathbf{d}_{e O}=\left(\mathbf{d}_{e 1}+\mathbf{d}_{e 2}+\mathbf{d}_{e 3}\right) / 3$

On the first sub-triangle $\Delta_{1}$ (triangle $0-1-2$ ), we now construct the linear approximation $\mathbf{u}_{e}^{\Delta_{1}}=\left[\begin{array}{llllll}u_{e}^{\Delta_{1}} & v_{e}^{\Delta_{1}} & w_{e}^{\Delta_{1}} & \beta_{e x}^{\Delta_{1}} & \beta_{e y}^{\Delta_{1}} & \beta_{e z}^{\Delta_{1}}\end{array}\right]^{T}$ by

$\mathbf{u}_{e}^{\Delta_{1}}=N_{1}^{\Delta_{1}}(\mathbf{x}) \mathbf{d}_{O}+N_{2}^{\Delta_{1}}(\mathbf{x}) \mathbf{d}_{1}+N_{3}^{\Delta_{1}}(\mathbf{x}) \mathbf{d}_{2}=\mathbf{N}_{e}^{\Delta_{1}}(\mathbf{x}) \mathbf{d}_{e}^{\Delta_{1}}$

where $\mathbf{d}_{e}^{\Delta_{1}}=\left[\begin{array}{lll}\mathbf{d}_{e O} & \mathbf{d}_{e 1} & \mathbf{d}_{e 2}\end{array}\right]^{T}$ is the vector of nodal degrees of freedom of the sub-triangle $\Delta_{1}$ and $\mathbf{N}_{e}^{\Delta_{1}}=\left[\begin{array}{lll}N_{1}^{\Delta_{1}} & N_{2}^{\Delta_{1}} & N_{3}^{\Delta_{1}}\end{array}\right]$ is the vector containing the linear shape functions in a natural coordinate defined by Eq. (18).

The membrane strain $\varepsilon_{e}^{m \Delta_{1}}$, the curvatures of the deflection $\boldsymbol{\kappa}_{e}^{\Delta_{1}}$ and the altered shear strains $\gamma_{e}^{\Delta_{1}}$ in the sub-triangle $\Delta_{1}$ are then obtained by

$\varepsilon_{e}^{m \Delta_{1}}=\underbrace{\left[\begin{array}{lll}\mathbf{r}_{1}^{\Delta_{1}} & \mathbf{r}_{2}^{\Delta_{1}} & \mathbf{r}_{3}^{\Delta_{1}}\end{array}\right]}_{\mathbf{r}_{e}^{\Delta_{1}}}\left[\begin{array}{l}\mathbf{d}_{O} \\ \mathbf{d}_{1} \\ \mathbf{d}_{2}\end{array}\right]=\mathbf{r}_{e}^{\Delta_{1}} \mathbf{d}_{e}^{\Delta_{1}}$ 

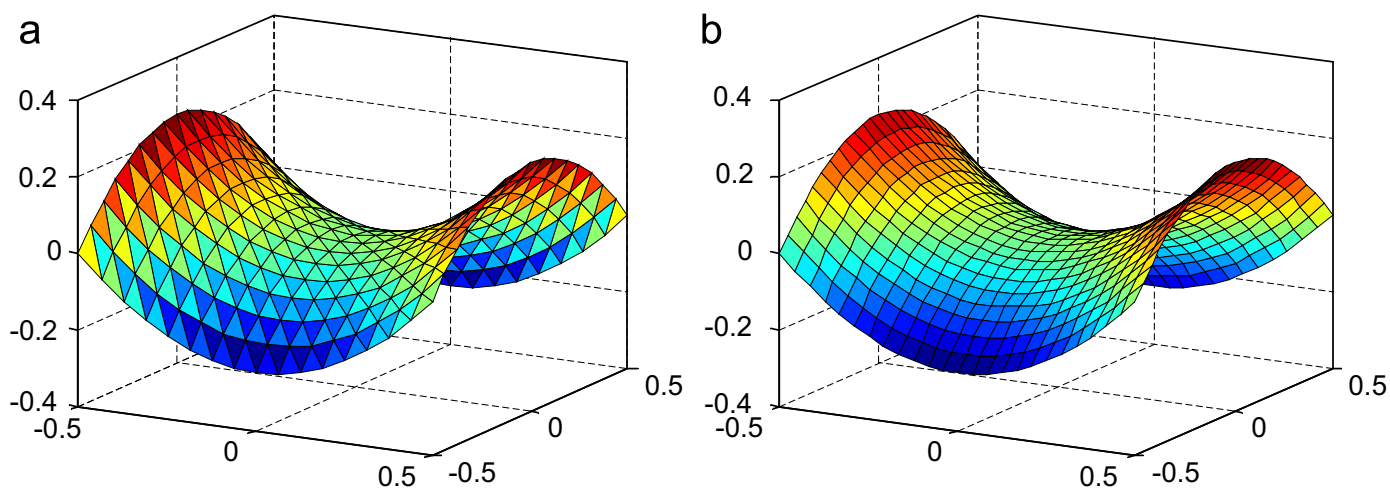

Fig. 15. Two discretizations of the partly clamped hyperbolic paraboloid shell structure using (a) triangular elements and (b) quadrilateral elements.

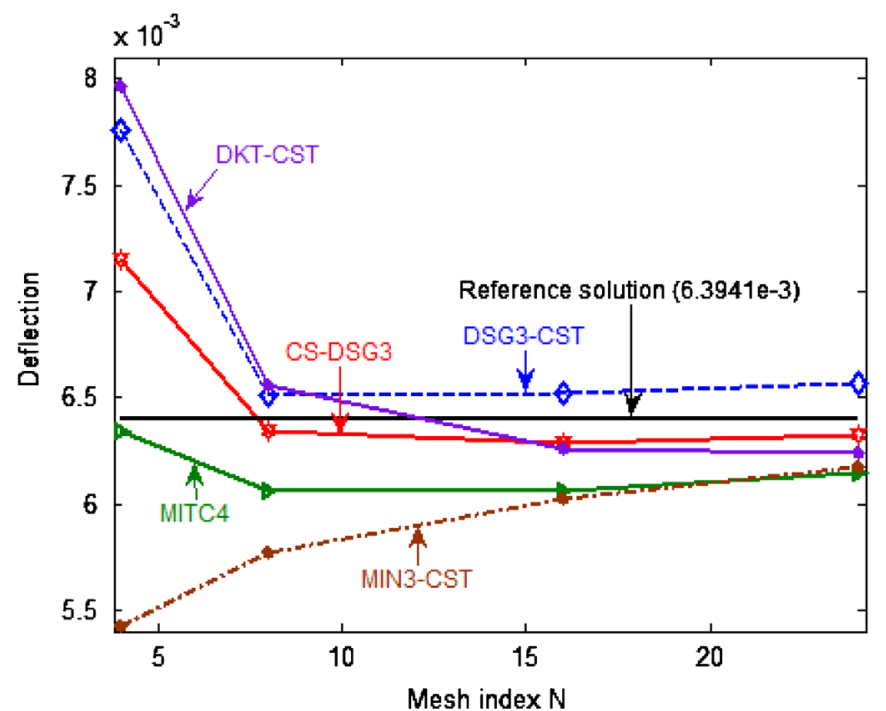

Fig. 16. Convergence of the vertical displacement at point $\mathrm{B}(x=L / 2, y=0)$ using structured meshes and different methods.

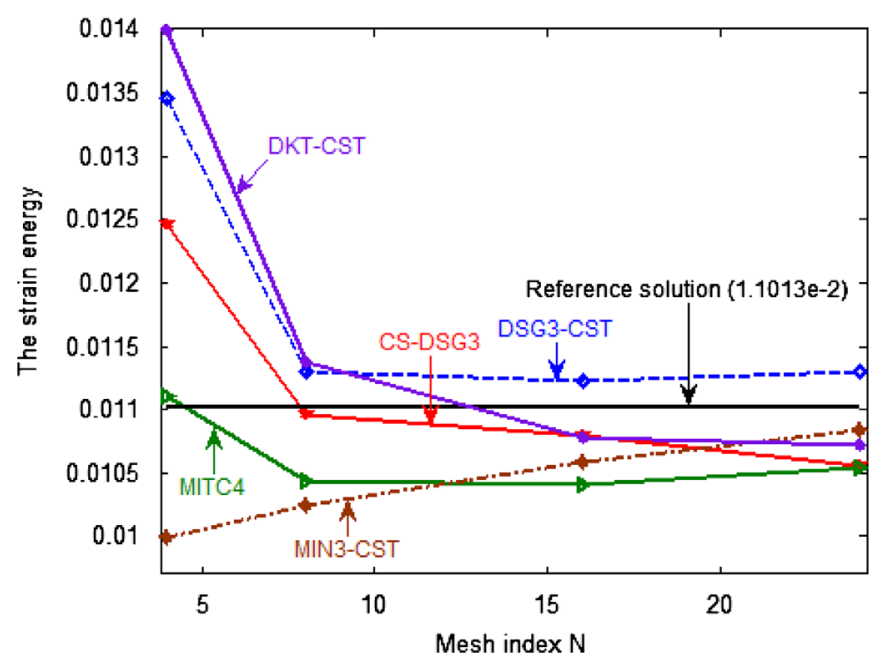

Fig. 17. Convergence of the strain energy of the partly clamped hyperbolic paraboloid shell structure obtained using structured meshes and different methods.

$\boldsymbol{\kappa}_{e}^{\Delta_{1}}=\underbrace{\left[\begin{array}{lll}\mathbf{b}_{1}^{\Delta_{1}} & \mathbf{b}_{2}^{\Delta_{1}} & \mathbf{b}_{3}^{\Delta_{1}}\end{array}\right]}_{\mathbf{b}_{e}^{\Delta_{1}}}\left[\begin{array}{l}\mathbf{d}_{e O} \\ \mathbf{d}_{e 1} \\ \mathbf{d}_{e 2}\end{array}\right]=\mathbf{b}_{e}^{\Delta_{1}} \mathbf{d}_{e}^{\Delta_{1}}$

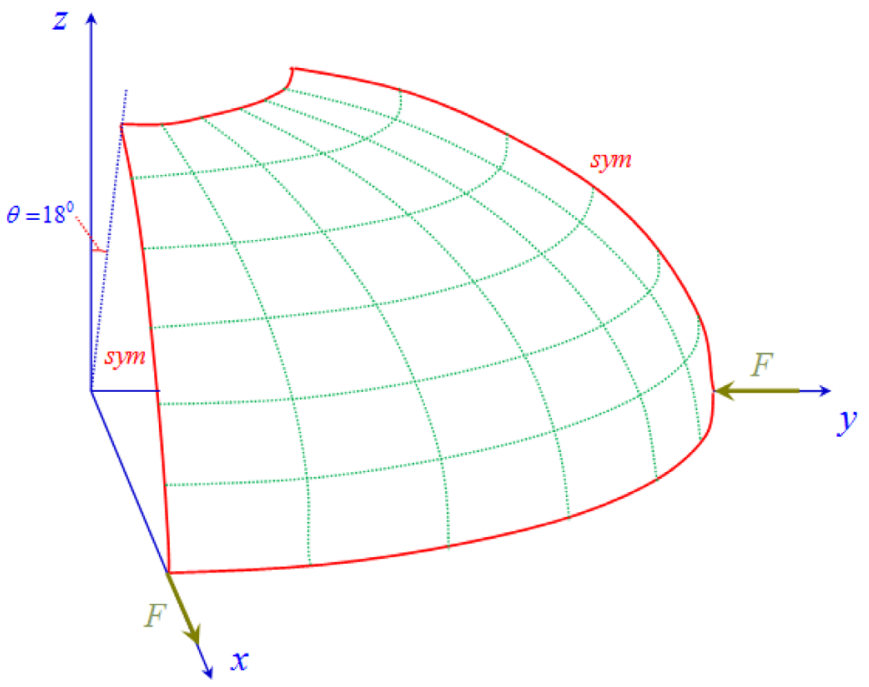

Fig. 18. Geometry of the hemispherical shell with an $18^{\circ}$ hole.

$\boldsymbol{\gamma}_{e}^{\Delta_{1}}=\underbrace{\left[\begin{array}{lll}\mathbf{s}_{1}^{\Delta_{1}} & \mathbf{s}_{2}^{\Delta_{1}} & \mathbf{s}_{3}^{\Delta_{1}}\end{array}\right]}_{\mathbf{s}_{e}^{\Delta_{1}}}\left[\begin{array}{l}\mathbf{d}_{e O} \\ \mathbf{d}_{e 1} \\ \mathbf{d}_{e 2}\end{array}\right]=\mathbf{s}_{e}^{\Delta_{1}} \mathbf{d}_{e}^{\Delta_{1}}$

where $\mathbf{r}_{e}^{\Delta_{1}}, \mathbf{b}_{e}^{\Delta_{1}}$ and $\mathbf{s}_{e}^{\Delta_{1}}$ are, respectively, computed similarly as the matrices R, B and $\mathbf{S}$ of the DSG3 by Eqs. (19), (20) and (23), but with two following changes: (1) the coordinates of three node $\mathbf{x}_{i}=\left[\begin{array}{ll}x_{i} & y_{i}\end{array}\right]^{T}, i=1,2,3$ are replaced by $\mathbf{x}_{0}, \mathbf{x}_{1}$ and $\mathbf{x}_{2}$, respectively; and (2) the area $A_{e}$ is replaced by the area $A_{\Delta_{1}}$ of sub-triangle $\Delta_{1}$.

Substituting $\mathbf{d}_{e O}$ in Eq. (30) into Eqs. (32)-(34), and then rearranging we obtain

$\boldsymbol{\varepsilon}_{e}^{m \Delta_{1}}=\underbrace{\left[\begin{array}{lll}\frac{1}{3} \mathbf{r}_{1}^{\Delta_{1}}+\mathbf{r}_{2}^{\Delta_{1}} & \frac{1}{3} \mathbf{r}_{1}^{\Delta_{1}}+\mathbf{r}_{3}^{\Delta_{1}} & \frac{1}{3} \mathbf{r}_{1}^{\Delta_{1}}\end{array}\right]}_{\mathbf{R}_{e}^{\Delta_{1}}}\left[\begin{array}{l}\mathbf{d}_{e 1} \\ \mathbf{d}_{e 2} \\ \mathbf{d}_{e 3}\end{array}\right]=\mathbf{R}_{e}^{\Delta_{1}} \mathbf{d}_{e}$
$\mathbf{\kappa}_{e}^{\Delta_{1}}=\underbrace{\left[\begin{array}{lll}\frac{1}{3} \mathbf{b}_{1}^{\Delta_{1}}+\mathbf{b}_{2}^{\Delta_{1}} & \frac{1}{3} \mathbf{b}_{1}^{\Delta_{1}}+\mathbf{b}_{3}^{\Delta_{1}} & \frac{1}{3} \mathbf{b}_{1}^{\Delta_{1}}\end{array}\right]}_{\mathbf{B}_{e}^{\Delta_{1}}}\left[\begin{array}{l}\mathbf{d}_{e 1} \\ \mathbf{d}_{e 2} \\ \mathbf{d}_{e 3}\end{array}\right]=\mathbf{B}_{e}^{\Delta_{1}} \mathbf{d}_{e}$

$\boldsymbol{\gamma}_{e}^{\Delta_{1}}=\underbrace{\left[\begin{array}{lll}\frac{1}{3} \mathbf{s}_{1}^{\Delta_{1}}+\mathbf{s}_{2}^{\Delta_{1}} & \frac{1}{3} \mathbf{s}_{1}^{\Delta_{1}}+\mathbf{s}_{3}^{\Delta_{1}} & \frac{1}{3} \mathbf{s}_{1}^{\Delta_{1}}\end{array}\right]}_{\mathbf{S}_{e}^{\Delta_{1}}}\left[\begin{array}{l}\mathbf{d}_{e 1} \\ \mathbf{d}_{e 2} \\ \mathbf{d}_{e 3}\end{array}\right]=\mathbf{S}_{e}^{\Delta_{1}} \mathbf{d}_{e}$

Similarly, by using the cyclic permutation, we easily obtain the membrane strain $\varepsilon_{e}^{m \Delta_{2}}, \varepsilon_{e}^{m \Delta_{3}}$, the curvatures of the deflection 
$\boldsymbol{\kappa}_{e}^{\Delta_{2}}, \boldsymbol{\kappa}_{e}^{\Delta_{3}}$ and the altered shear strains $\gamma_{e}^{\Delta_{2}}, \gamma_{e}^{\Delta_{3}}$ for the second subtriangle $\Delta_{2}$ and third sub-triangle $\Delta_{3}$, respectively.

Now, applying the cell-based strain smoothing operation in the CS-FEM [17,18], the constant membrane strains $\varepsilon_{e}^{m \Delta_{1}}, \varepsilon_{e}^{m \Delta_{2}}, \varepsilon_{e}^{m \Delta_{3}}$, the constant bending strains $\boldsymbol{\kappa}_{e}^{\Delta_{1}}, \boldsymbol{\kappa}_{e}^{\Delta_{2}}, \boldsymbol{\kappa}_{e}^{\Delta_{3}}$ and constant shear strains $\gamma_{e}^{\Delta_{1}}, \gamma_{e}^{\Delta_{2}}, \gamma_{e}^{\Delta_{3}}$ are, respectively, used to create a smoothed element membrane strain $\tilde{\boldsymbol{\varepsilon}}_{e}^{m}$, a smoothed element bending strain $\tilde{\boldsymbol{\kappa}}_{e}$ and a smoothed element shear strain $\tilde{\gamma}_{e}$ on the triangular element $\Omega_{e}$, such as

$\tilde{\varepsilon}_{e}^{m}=\int_{\Omega_{e}} \varepsilon^{m} \Phi_{e}(\mathbf{x}) \mathrm{d} \Omega=\sum_{i=1}^{3} \varepsilon_{e}^{m \Delta_{i}} \int_{\Delta_{i}} \Phi_{e}(\mathbf{x}) \mathrm{d} \Omega$

$\tilde{\mathbf{\kappa}}_{e}=\int_{\Omega_{e}} \mathbf{\kappa}^{h} \Phi_{e}(\mathbf{x}) \mathrm{d} \Omega=\sum_{i=1}^{3} \mathbf{\kappa}_{e}^{\Delta_{i}} \int_{\Delta_{i}} \Phi_{e}(\mathbf{x}) \mathrm{d} \Omega$

$\tilde{\gamma}_{e}=\int_{\Omega_{e}} \boldsymbol{\gamma}^{h} \Phi_{e}(\mathbf{x}) \mathrm{d} \Omega=\sum_{i=1}^{3} \gamma_{e}^{\Delta_{i}} \int_{\Delta_{i}} \Phi_{e}(\mathbf{x}) \mathrm{d} \Omega$

where $\Phi_{e}(\mathbf{x})$ is a given smoothing function that satisfies at least unity property $\int_{\Omega_{e}} \Phi_{e}(\mathbf{x}) \mathrm{d} \Omega=1$. In this paper, we use the Heavisidetype constant smoothing function

$\Phi_{e}(\mathbf{x})=\left\{\begin{array}{cc}1 / A_{e} & \mathbf{x} \in \Omega_{e} \\ 0 & \mathbf{x} \notin \Omega_{e}\end{array}\right.$

where $A_{e}$ is the area of the triangular element, the smoothed element membrane strain $\tilde{\varepsilon}_{e}^{m}$, the smoothed element bending strain $\tilde{\boldsymbol{\kappa}}_{e}$ and the smoothed element shear strain $\tilde{\gamma}_{e}$ in Eqs. (38)(40) become

$\tilde{\boldsymbol{\varepsilon}}_{e}^{m}=\tilde{\mathbf{R}}_{e} \mathbf{d}_{e} ; \quad \tilde{\mathbf{\kappa}}_{e}=\tilde{\mathbf{B}}_{e} \mathbf{d}_{e} ; \quad \tilde{\boldsymbol{\gamma}}_{e}=\tilde{\mathbf{S}}_{e} \mathbf{d}_{e}$

where $\tilde{\mathbf{R}}_{e}, \tilde{\mathbf{B}}_{e}$ and $\tilde{\mathbf{S}}_{e}$ are, respectively, the smoothed membrane gradient matrix, smoothed bending gradient matrix and smoothed shear strain gradient matrix given by

$\tilde{\mathbf{R}}_{e}=\frac{1}{A_{e}} \sum_{i=1}^{3} A_{\Delta_{i}} \mathbf{R}_{e}^{\Delta_{i}} ; \quad \tilde{\mathbf{B}}_{e}=\frac{1}{A_{e}} \sum_{i=1}^{3} A_{\Delta_{i}} \mathbf{B}_{e}^{\Delta_{i}} ; \quad \tilde{\mathbf{S}}_{e}=\frac{1}{A_{e}} \sum_{i=1}^{3} A_{\Delta_{i}} \mathbf{S}_{e}^{\Delta_{i}}$

Therefore the global stiffness matrix of the CS-DSG3 are assembled by

$\tilde{\mathbf{K}}=\sum_{e=1}^{N_{e}} \tilde{\mathbf{K}}_{e}$

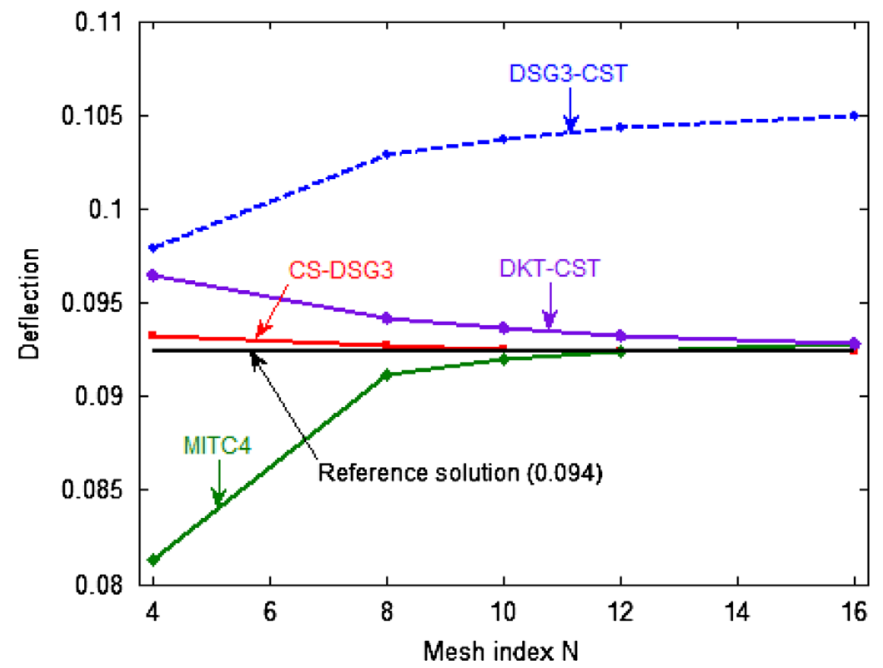

Fig. 20. Convergence of the radial deflection coincident at point load of the hemispherical shell with an $18^{\circ}$ hole using structured meshes and different methods.

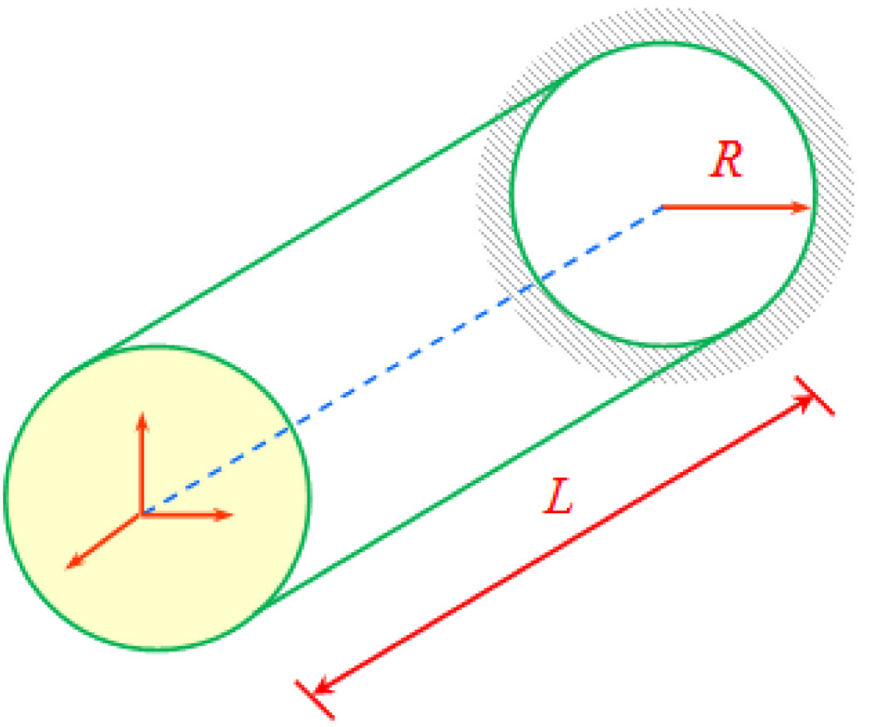

Fig. 21. Geometry of the cylindrical shell with clamped at one edge and free at the other

b

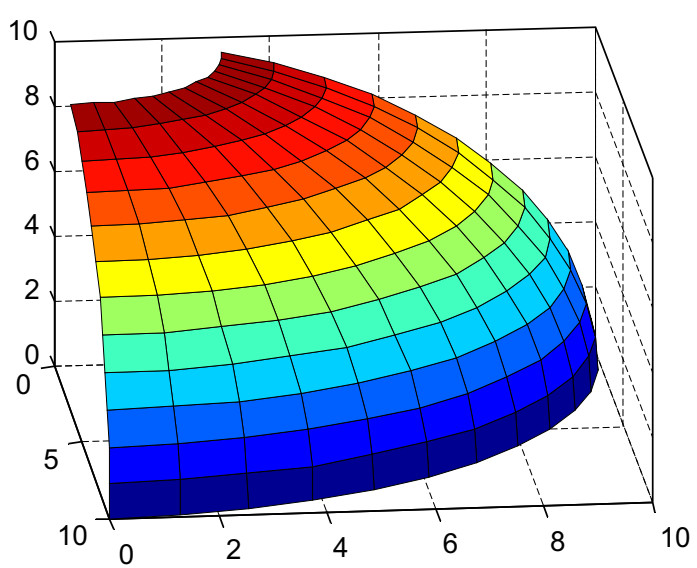

Fig. 19. Two discretizations of the hemispherical shell with an $18^{\circ}$ hole using (a) triangular elements and (b) quadrilateral elements. 
where $\tilde{\mathbf{K}}_{e}$ is the smoothed element stiffness given by

$$
\begin{aligned}
\tilde{\mathbf{K}}_{e} & =\mathbf{T}^{T}\left(\int_{\Omega_{e}} \tilde{\mathbf{R}}^{T} \mathbf{D}^{m} \tilde{\mathbf{R}} \mathrm{d} \Omega+\int_{\Omega_{e}} \tilde{\mathbf{B}}^{T} \mathbf{D}^{b} \tilde{\mathbf{B}} \mathrm{d} \Omega+\int_{\Omega_{e}} \tilde{\mathbf{S}}^{T} \mathbf{D}^{s} \tilde{\mathbf{S}} \mathrm{d} \Omega\right) \mathbf{T} \\
& =\mathbf{T}^{T} \underbrace{\left(\tilde{\mathbf{R}}^{T} \mathbf{D}^{m} \tilde{\mathbf{R}} A_{e}+\tilde{\mathbf{B}}^{T} \mathbf{D}^{b} \tilde{\mathbf{B}} A_{e}+\tilde{\mathbf{S}}^{T} \mathbf{D}^{s} \tilde{\mathbf{S}} A_{e}\right)}_{\tilde{\mathbf{k}}_{e}} \mathbf{T}
\end{aligned}
$$$$
=\mathbf{T}^{T} \tilde{\mathbf{k}}_{e} \mathbf{T}
$$

in which $\tilde{\mathbf{k}}_{e}$ is the smoothed element stiffness matrix computed in the local coordinate system.

From Eqs. (43) and (45), it is seen that the element stiffness matrix in the CS-DSG3 does not depend on the sequence of node numbers, and hence the solution of CS-DSG3 is always stable when the sequence of node numbers changes. Also note that the rank of the CS-DSG3 element is similar to that of the DSG3 element and hence the kinematic stability of the CS-DSG3 element is ensured. Only six eigenvalues are always zero (corresponding to the rigid body modes of the element) for various element shapes of very thin and thick shells, and the CS-DSG3 element has no spurious zero-energy modes as shown in various numerical examples in Section 4.

\section{Numerical results}

In this section, various numerical examples are performed to show the accuracy and stability of the proposed CS-DSG3 compared to the analysis solutions. The stabilized parameter $\alpha$ in Eq. (29) in the CS-DSG3 is fixed at 0.1 for both static analysis and free vibration analysis. For comparison, several others flat shell elements such as DSG3 [15], DKT [28], MIN3 [29] and MITC4 $[13,30]$ have also been implemented in our package. The membrane element used here in others triangular flat shell elements is the constant strain triangular element (CST), and hence three triangular flat shell elements used for comparion are abbreviated as DSG3-CST, DKT-CST and MIN3-CST, respectively.
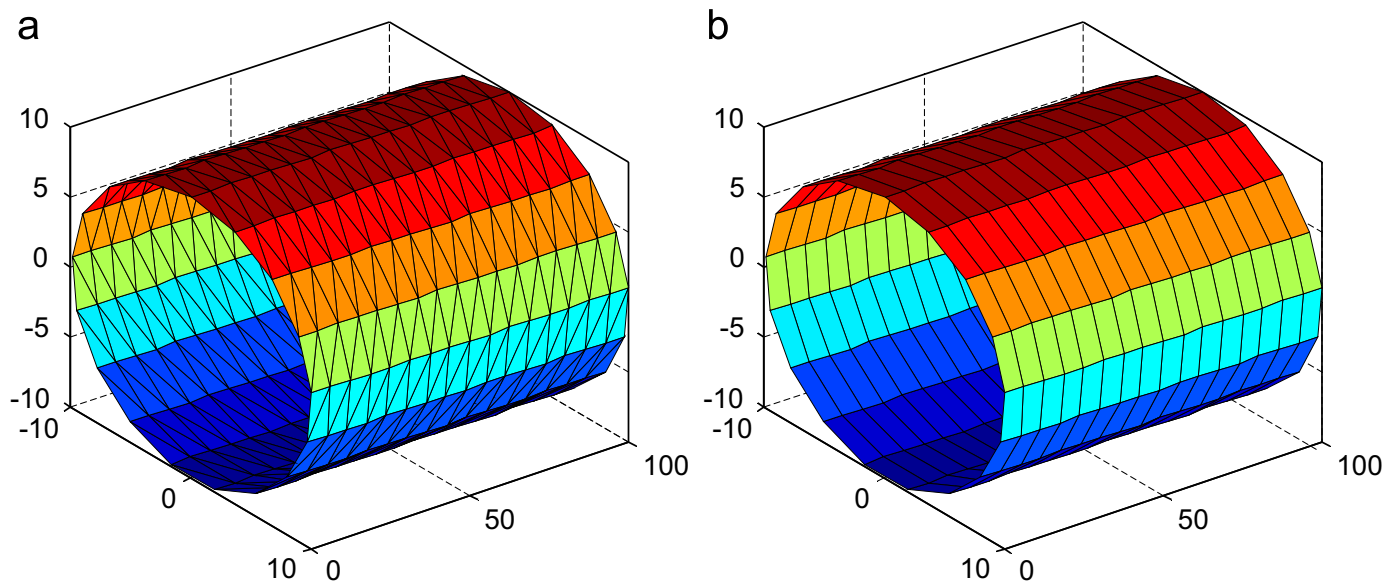

Fig. 22. Two discretizations of the cylindrical shell using (a) triangular elements and (b) quadrilateral elements.

Table 1

\begin{tabular}{|c|c|c|c|c|c|c|c|c|c|}
\hline \multirow[t]{2}{*}{ Meshing } & \multirow[t]{2}{*}{ Method } & \multicolumn{8}{|l|}{ Mode } \\
\hline & & 1 & 2 & 3 & 4 & 5 & 6 & 7 & 8 \\
\hline \multirow[t]{5}{*}{$8 \times 8$} & DKT-CST & 1.4718 & 1.4891 & 2.3898 & 2.4023 & 3.0964 & 3.1739 & 6.1941 & 6.5235 \\
\hline & MITC4 & 1.339 & 1.3442 & 2.2058 & 2.2137 & 4.1676 & 4.2499 & 5.3046 & 5.3752 \\
\hline & MIN3-CST & 1.8052 & 1.8111 & 2.3933 & 2.4063 & 3.6943 & 3.8189 & 6.7842 & 6.8582 \\
\hline & DSG3-CST & 1.5426 & 1.5598 & 2.3898 & 2.4032 & 4.2348 & 4.3217 & 6.7091 & 6.8252 \\
\hline & CS-DSG3 & 1.4555 & 1.4754 & 2.3898 & 2.402 & 2.9621 & 3.0064 & 5.7479 & 5.9713 \\
\hline \multirow[t]{5}{*}{$12 \times 12$} & DKT-CST & 1.2529 & 1.2562 & 2.3032 & 2.3059 & 2.6487 & 2.6616 & 4.5491 & 4.5781 \\
\hline & MITC4 & 1.1875 & 1.1891 & 2.2128 & 2.2144 & 2.9256 & 2.9361 & 4.0134 & 4.0247 \\
\hline & MIN3-CST & 2.0066 & 2.0141 & 2.3121 & 2.3147 & 4.0048 & 4.0436 & 5.5557 & 5.5561 \\
\hline & DSG3-CST & 1.2811 & 1.2847 & 2.3032 & 2.306 & 2.9888 & 2.9977 & 4.7858 & 4.8139 \\
\hline & CS-DSG3 & 1.2493 & 1.2531 & 2.3032 & 2.3059 & 2.6207 & 2.6281 & 4.5391 & 4.5708 \\
\hline \multirow[t]{5}{*}{$16 \times 16$} & DKT-CST & 1.1758 & 1.1768 & 2.2692 & 2.2701 & 2.4689 & 2.4727 & 3.8839 & 3.8928 \\
\hline & MITC4 & 1.1377 & 1.1382 & 2.2157 & 2.2162 & 2.5984 & 2.6012 & 3.586 & 3.5903 \\
\hline & MIN3-CST & 2.2858 & 2.2865 & 2.3986 & 2.4033 & 4.666 & 4.6837 & 5.4642 & 5.4708 \\
\hline & DSG3-CST & 1.191 & 1.1921 & 2.2692 & 2.2702 & 2.6317 & 2.6339 & 4.0026 & 4.0119 \\
\hline & CS-DSG3 & 1.1747 & 1.1759 & 2.2692 & 2.2701 & 2.4632 & 2.4653 & 3.8827 & 3.8926 \\
\hline \multirow[t]{5}{*}{$20 \times 20$} & DKT-CST & 1.1396 & 1.14 & 2.2523 & 2.2527 & 2.3829 & 2.3844 & 3.5928 & 3.5963 \\
\hline & MITC4 & 1.1148 & 1.115 & 2.217 & 2.2172 & 2.4592 & 2.4603 & 3.4034 & 3.4053 \\
\hline & MIN3-CST & 2.279 & 2.2792 & 2.8451 & 2.8481 & 5.4428 & 5.4522 & 5.5335 & 5.535 \\
\hline & DSG3-CST & 1.1492 & 1.1496 & 2.2525 & 2.2528 & 2.4789 & 2.4797 & 3.6647 & 3.6685 \\
\hline & CS-DSG3 & 1.1392 & 1.1397 & 2.2524 & 2.2527 & 2.3829 & 2.3837 & 3.5926 & 3.5965 \\
\hline Leissa [35] & & 1.1094 & & 2.4578 & & & & & \\
\hline RSQ20 [8] & & 1.0915 & 1.0915 & 2.2366 & 2.2366 & & & & \\
\hline RSQ24 [8] & & 1.1006 & 1.1006 & 2.2374 & 2.2773 & & & & \\
\hline
\end{tabular}

Convergence of eight lowest frequency parameters $\lambda=100 \omega R \sqrt{\rho\left(1-\nu^{2}\right) / E}$ of the CFFF cylindrical shell. 


\subsection{Static analysis}

4.1.1. Cylindrical shell under central point load-pinched cylinder with diaphragm

We now consider a pinched cylindrical shell supported at each end by rigid diaphragm and subjected to a point load $P=1$ at the center of the cylindrical surface as shown in Fig. 6 . The geometric dimensions of the pinched cylinder are given by the length $L=600$, the radius $R=300$ and the thickness $t=3$. The material properties are given by Poisson's ratio $\Omega_{e}$ and Young's modulus $E=3 \times 10^{6}$. Due to its symmetry, only one eighth of the cylinder is modeled. Six uniform discretizations $N \times N$ of shell with $N=8,12,16,20,24$ and 28 are used and two discretizations $16 \times 16$ using triangular and quadrilateral elements are plotted in Fig. 7.

Fig. 8 shows the convergence of the central deflection at point A obtained using structured meshes and different methods.
The reference solution obtained from [31] is $1.8248 \times 10^{-5}$. It is seen that with the same degree of freedoms (DOFs), the results of the CS-DSG3 are better than those of almost methods and only worse than those of the DKT-CST in some coarse meshes. Also note that, the CS-DSG3 shows the best accuracy for the fine meshes.

Fig. 9 shows the convergence of the strain energy obtained using structured meshes and different methods. The results again confirm the comments obtained for the central deflection at point A shown in Fig. 8.

\subsubsection{Cylindrical shell under uniform load-Scordelis-Lo roof}

We now consider a cylindrical shell roof known as the ScordelisLo roof in which two curved edges are supported by rigid diaphragms $(u=w=0)$, and the other two edges are free as shown in Fig. 10. The Scordelis-Lo roof is subjected to the self-weight $q=90$ per unit area in the $z$-direction and has the geometric dimensions
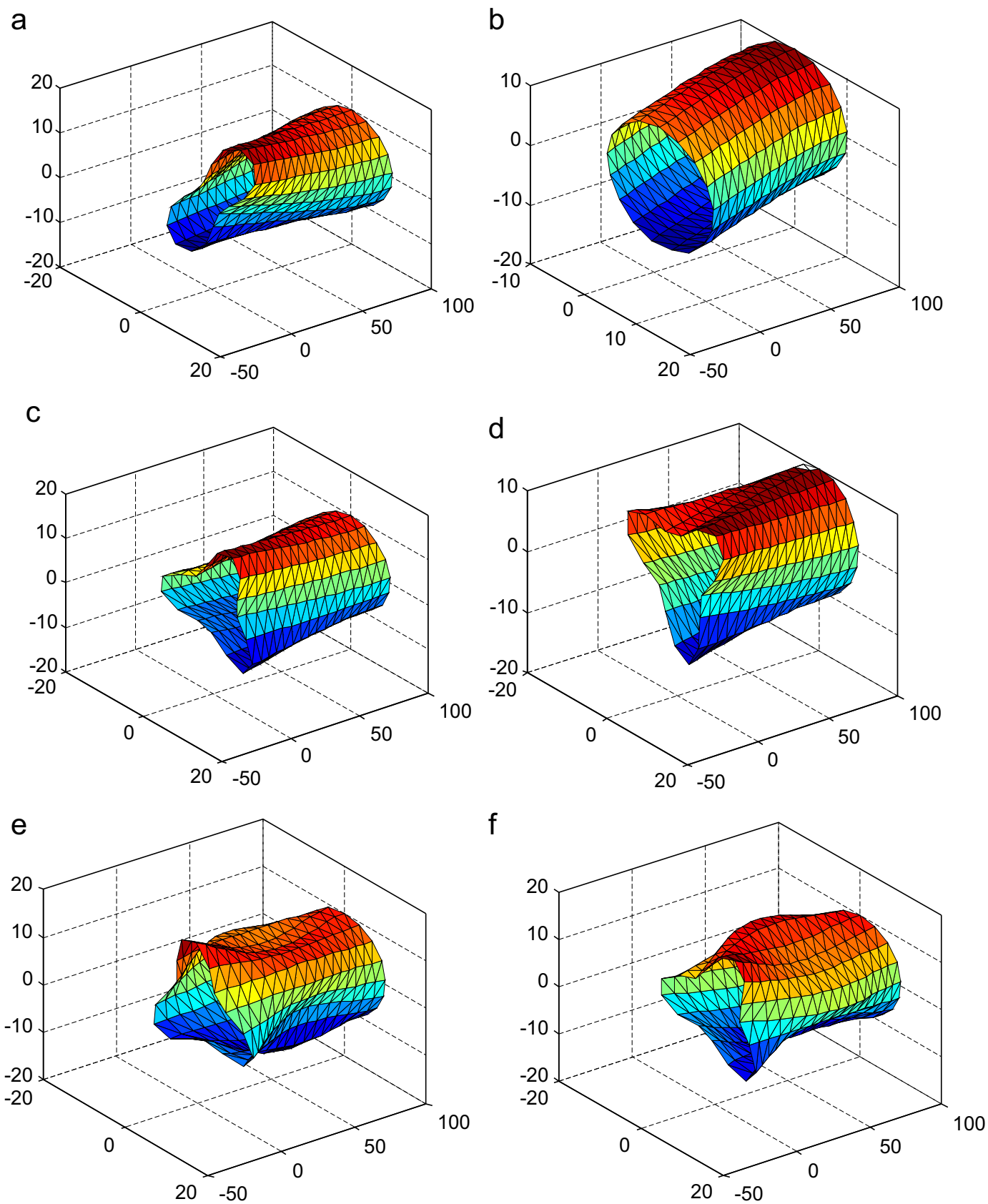

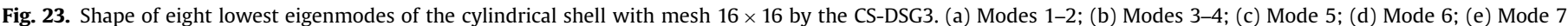
and (f) Mode 8. 
given by the length $L=50$, the radius $R=25$ and the thickness $t=0.25$. The material properties are given by Poisson's ratio $v=0.0$ and Young's modulus $E=4.32 \times 10^{8}$. This example was first modeled by Scordelis and Lo [32] who gave the mid-side vertical displacement at point $A$ is 0.3086 . However, many finite elements converge to a slightly smaller value, and hence Macneal and Harder [33] suggested to use the value of 0.3024 for testing. Due to its symmetry, only a quarter of the cylinder shell is modeled. Five uniform discretizations $N \times N$ of shell with $N=4,8,10,12$ and 16 are used and two discretizations $16 \times 16$ using triangular and quadrilateral elements are plotted in Fig. 11.

Fig. 12 shows the convergence of the mid-side vertical displacement at point A obtained using structured meshes and different methods. It is seen that with the same degree of freedoms (DOFs), the results of the CS-DSG3 are better than those of almost methods and only worse than those of the MITC4 in some coarse meshes. Also note that, the CS-DSG3 shows the fastest convergence to the reference solution for the fine meshes.

Fig. 13 shows the convergence of the strain energy obtained using structured meshes and different methods. The results again confirm the comments obtained the mid-side vertical displacement at point A shown in Fig. 12.

\subsubsection{Partly clamped hyperbolic paraboloid}

We now consider a hyperbolic paraboloid shell structure with the geometric equation $z=x^{2}-y^{2}, x \in[-0.5,0.5]$ and $y \in[-0.5,0.5]$ as shown in Fig. 14. The hyperbolic paraboloid shell structure is clamped along one side and subjected to the self-weight $q=8000 \mathrm{~kg} / \mathrm{m}^{3}$ in the $z$-direction. The geometric dimensions are given by the length $L=1 \mathrm{~m}$ and the thickness $t=0.001 \mathrm{~m}$. The material properties are given by Poisson's ratio $v=0.3$ and Young's modulus $E=2 \times 10^{11} \mathrm{~N} / \mathrm{m}^{2}$. The reference values [34] for the total strain energy is $1.1013 \times 10^{-2} \mathrm{Nm}^{2}$ and the vertical displacement at point $\mathrm{B}(x=L / 2, y=0)$ is $-6.3941 \times 10^{-3} \mathrm{~m}$. Four uniform discretizations $N \times N$ of shell with $N=4,8,16$ and 24 are used and two discretizations $16 \times 16$ using triangular and quadrilateral elements are plotted in Fig. 15.

Fig. 16 shows the convergence of the vertical displacement at point $\mathrm{B}(x=L / 2, y=0)$ obtained using structured meshes and different methods. It is seen that the CS-DSG3 shows remarkably excellent performance compared to the other methods.

Fig. 17 shows the convergence of the strain energy obtained using structured meshes and different methods. The results again confirm the comments obtained the vertical displacement at point $\mathrm{B}(x=L / 2, y=0)$ shown in Fig. 16 .

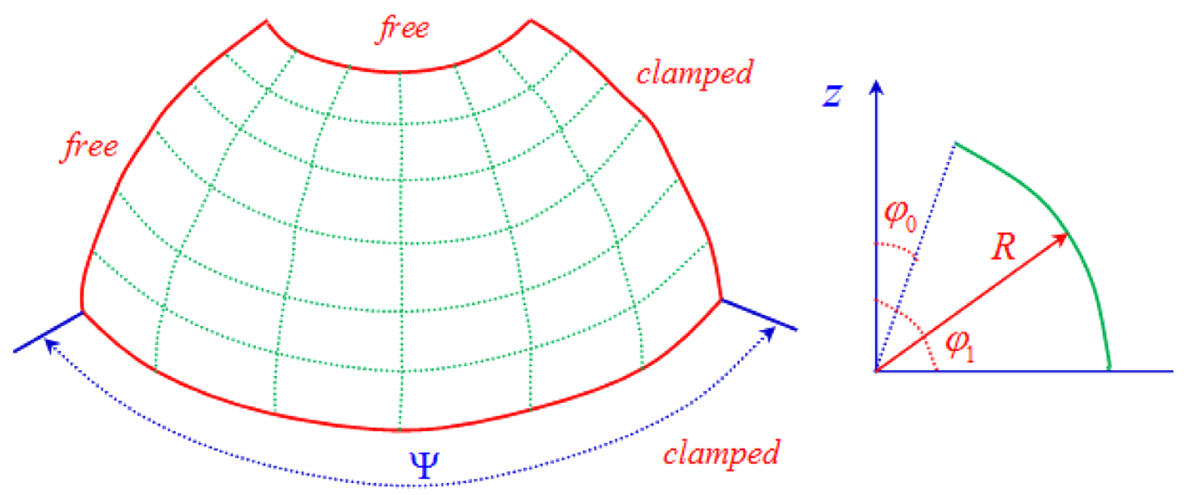

Fig. 24. Geometry of the hemispherical panel with clamped at two edges and free at two other edges.

a

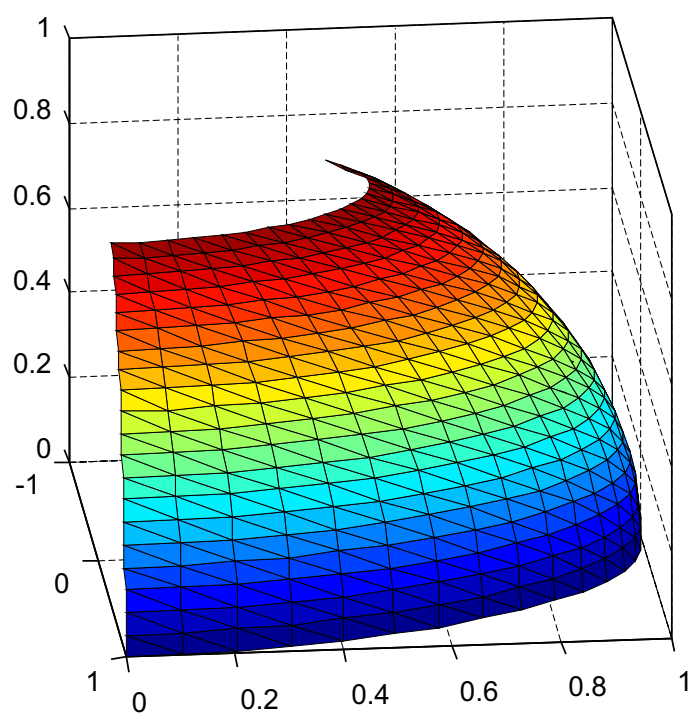

b

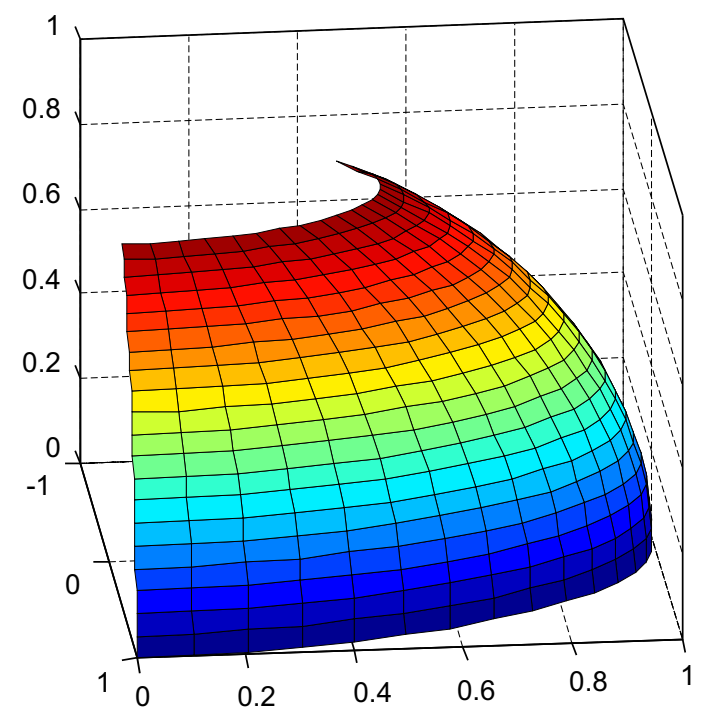

Fig. 25. Two discretizations of the hemispherical panel using (a) triangular elements and (b) quadrilateral elements. 


\subsubsection{Hemispherical shell}

We now consider a hemispherical shell with an $18^{\circ}$ hole subjected to two point loads $F=1 \mathrm{~N}$ antisymmetrically as shown in Fig. 18. This benchmark problem aims to test the ability of an element to handle rigid body rotations about normals to the shell surface. The geometric dimensions are given by the radius $R=10 \mathrm{~m}$ and the thickness $t=0.04 \mathrm{~m}$. The material properties are given by Poisson's ratio $v=0.3$ and Young's modulus $E=6.825 \times 10^{7} \mathrm{~N} / \mathrm{m}^{2}$. The reference values [33] for the radial deflection coincident at point load is $0.0924 \mathrm{~m}$. Five uniform discretizations $N \times N$ of shell with $N=4,8,10,12$ and 16 are used and two discretizations $12 \times 12$ using triangular and quadrilateral elements are plotted in Fig. 19.

Fig. 20 shows the convergence of the radial deflection coincident at point load obtained using structured meshes and different methods. It is seen that the CS-DSG3 shows excellent performance compared to the other methods.

\subsection{Free vibration analysis}

In this section, we will examine the accuracy of the CS-DSG3 element in solving for natural frequencies of various shell structures. The shell may have different boundary conditions such as free (F), simply (S) supported or clamped (C) edges.

\subsubsection{A cylindrical shell with clamped-free ends (CFFF)}

We now analyze the natural frequencies of a cylindrical shell with clamped at one edge and free at the other as shown in Fig. 21. The geometric and material properties are given by length to radius ratios $L / R=10$, radius to thickness ratios $R / t=100$, elastic modulus $E=2.1 \times 10^{11} \mathrm{~N} / \mathrm{m}^{2}$, Poisson ratio $\nu=0.3$ and mass density $\rho=7800 \mathrm{~kg} / \mathrm{m}^{3}$. Four uniform discretizations $N \times N$ of shell with $N=8,12,16$ and 20 are used and two discretizations $12 \times 12$ using triangular and quadrilateral elements are plotted in Fig. 22. The natural frequency parameters $\lambda=100 \omega R \sqrt{\rho\left(1-\nu^{2}\right) / E}$ is used to illustrate the numerical results.
Table 1 shows eight lowest modes by the CS-DSG3 and various methods. The results are also compared with the analytical solution by Leissa [35] and numerical results of the RSQ20 and RSQ24 quadrilateral elements [8]. It is observed that the results of CS-DSG3 converge well to the reference solution by Leissa [35] and reference numeral results of RSQ20 and RSQ24 [8]. It is also seen that the CS-DSG3 is better than the DSG3-CST, MIN3-CST, DKTCST and a good competitor to the MITC4. Fig. 23 plots the shape of eight lowest eigenmodes of the cylindrical shell with mesh $16 \times 16$ by the CS-DSG3.

\subsubsection{Hemispherical panel CCFF}

Let us now consider a hemispherical panel as shown in Fig. 24 with radius $R=1 \mathrm{~m}$, thickness $t=0.1 \mathrm{~m}, \varphi_{0}=30^{\circ}, \varphi_{1}=90^{\circ}, \psi=120^{\circ}$.

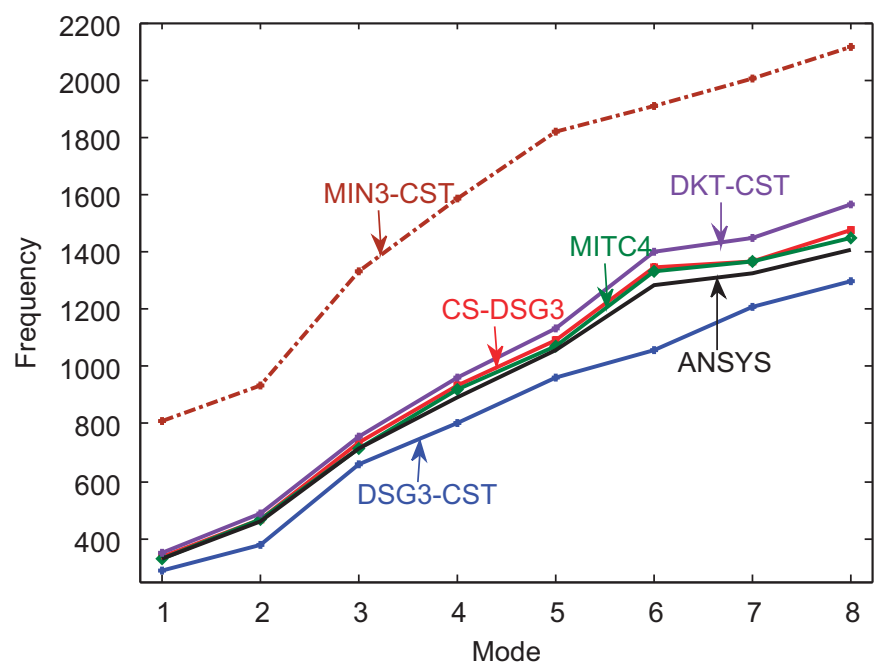

Fig. 26. Eight lowest frequencies of the hemispherical panel discretized by a uniform mesh $12 \times 12$.

Table 2

Convergence of eight lowest frequencies of the hemispherical panel CCFF.

\begin{tabular}{|c|c|c|c|c|c|c|c|c|c|}
\hline \multirow[t]{2}{*}{ Meshing } & \multirow[t]{2}{*}{ Method } & \multicolumn{8}{|l|}{ Mode } \\
\hline & & 1 & 2 & 3 & 4 & 5 & 6 & 7 & 8 \\
\hline \multirow[t]{5}{*}{$8 \times 8$} & DKT-CST & 359.3 & 496.4 & 777.5 & 1009 & 1178 & 1465.2 & 1506.5 & 1671.3 \\
\hline & MITC4 & 332.5 & 471.3 & 729 & 950.7 & 1102.9 & 1392.7 & 1420.6 & 1520.9 \\
\hline & MIN3-CST & 771 & 890.7 & 1273.8 & 1558.9 & 1810 & 1932.6 & 1980.5 & 2043.1 \\
\hline & DSG3-CST & 288.8 & 366.6 & 663.9 & 808.5 & 993.6 & 1059.2 & 1298.8 & 1344.9 \\
\hline & CS-DSG3 & 346.3 & 472.4 & 750.6 & 983.4 & 1136.4 & 1378.1 & 1450.1 & 1585.1 \\
\hline \multirow[t]{5}{*}{$12 \times 12$} & DKT-CST & 350.6 & 487.1 & 755.1 & 960.8 & 1130.8 & 1398.7 & 1451.3 & 1566.9 \\
\hline & MITC4 & 328.9 & 464.5 & 715.7 & 917.3 & 1073.4 & 1334.5 & 1367 & 1447.4 \\
\hline & MIN3-CST & 809.4 & 931.3 & 1329.9 & 1586 & 1817.2 & 1906.1 & 2004 & 2116.4 \\
\hline & DSG3-CST & 287.5 & 374.4 & 655.8 & 802.7 & 963.3 & 1057.2 & 1207.9 & 1296.6 \\
\hline & CS-DSG3 & 338.3 & 467 & 731.6 & 933.0 .3 & 1089.6 & 1346.1 & 1368.2 & 1475.6 \\
\hline \multirow[t]{5}{*}{$16 \times 16$} & DKT-CST & 346.4 & 483.2 & 745 & 942 & 1113.3 & 1368.4 & 1429.8 & 1529.5 \\
\hline & MITC4 & 328 & 462.6 & 711.5 & 906.1 & 1064.4 & 1311.5 & 1349.5 & 1424.1 \\
\hline & MIN3-CST & 831.2 & 954.8 & 1359.6 & 1612.1 & 1851.9 & 1911.7 & 2039.4 & 2175.1 \\
\hline & DSG3-CST & 286.2 & 377.3 & 652.1 & 797.8 & 951.4 & 1061.1 & 1177.6 & 1257.1 \\
\hline & CS-DSG3 & 334.4 & 464.1 & 722.4 & 913.7 & 1071.8 & 1329.1 & 1331.4 & 1436.2 \\
\hline \multirow[t]{5}{*}{$20 \times 20$} & DKT-CST & 344.2 & 481.2 & 739.7 & 932.8 & 1105 & 1352.6 & 1419.3 & 1512.8 \\
\hline & MITC4 & 327.7 & 461.8 & 709.7 & 901.1 & 1060.7 & 1300.7 & 1342 & 1414.2 \\
\hline & MIN3-CST & 844.6 & 969.8 & 1376 & 1630.8 & 1882.5 & 1925.1 & 2068.3 & 2219.4 \\
\hline & DSG3-CST & 285.4 & 378.9 & 650.2 & 795.1 & 946 & 1063.8 & 1164.1 & 1237.6 \\
\hline & CS-DSG3 & 332.2 & 462.4 & 717.3 & 904.1 & 1063.2 & 1309.2 & 1323.1 & 1417.6 \\
\hline Abaqus & & 326.94 & 459.01 & 706.98 & 884.09 & 1047.6 & 1270.8 & 1309.2 & 1383.7 \\
\hline Ansys & & 328.48 & 460.89 & 710.52 & 893.51 & 1056.1 & 1285.2 & 1328 & 1404 \\
\hline Nastran & & 328.69 & 460.93 & 711.09 & 892.71 & 1055.8 & 1282.4 & 1325.9 & 1401.9 \\
\hline Straus & & 327.28 & 458.54 & 706.64 & 888.86 & 1049.5 & 1278.9 & 1313.9 & 1395.5 \\
\hline GDQ & & 327.39 & 458.58 & 705.71 & 885.18 & 1046.6 & 1270.7 & 1305.1 & 1382.8 \\
\hline
\end{tabular}


The material parameters are given by Young's modulus $E=2.1 \times$ $10^{11} \mathrm{~Pa}$, Poisson's ratio $\nu=0.3$, mass density $\rho=7800 \mathrm{~kg} / \mathrm{m}^{3}$. Four uniform discretizations $N \times N$ of shell with $N=8,12,16$ and 20 are used and two discretizations $12 \times 12$ using triangular and quadrilateral elements are plotted in Fig. 25.

Eight lowest modes by the CS-DSG3 and various methods are shown in Table 2 and plotted in Fig. 26 with mesh $12 \times 12$. The results are also compared with the numerical solutions of the Generalized Differential Quadrature (GDQ) method [36] and the reference results derived from commercial software packages such as Abaqus, Ansys, Nastran, Straus found in Ref [36]. It is again observed that the results of CS-DSG3 converge well to the reference solutions of the GDQ [36] and of commercial software packages such as Abaqus, Ansys, Nastran, Straus [36]. It is also seen that the CS-DSG3 is better than the DSG3CST, MIN3-CST, DKT-CST and a good competitor to the MITC4. Fig. 27 plots the shape of six lowest eigenmodes of the hemispherical panel with mesh $16 \times 16$ by the CS-DSG3.

\section{Conclusions}

A cell-based smoothed discrete shear gap method (CS-DSG3) for static and free vibration analyses of Reissner-Mindlin shells is formulated by combining the cell-based strain smoothing technique with the discrete shear gap method (DSG3) using three-node triangular elements. In the CS-DSG3, each triangular element will be divided into three sub-triangles, and in each sub-triangle, the stabilized DSG3 is used to compute the strains and to avoid the transverse shear locking. Then the strain smoothing technique on whole of the triangular element is used to smooth the strains on these three sub-triangles. The CS-DSG3 hence not only overcomes the drawback of the DSG3 which depends on the sequence of node numbers of elements, but also improves the accuracy as well as the stability of the DSG3. The numerical examples demonstrated that the CS-DSG3 is free of shear locking and achieves the high accuracy compared to others existing flat shell elements.
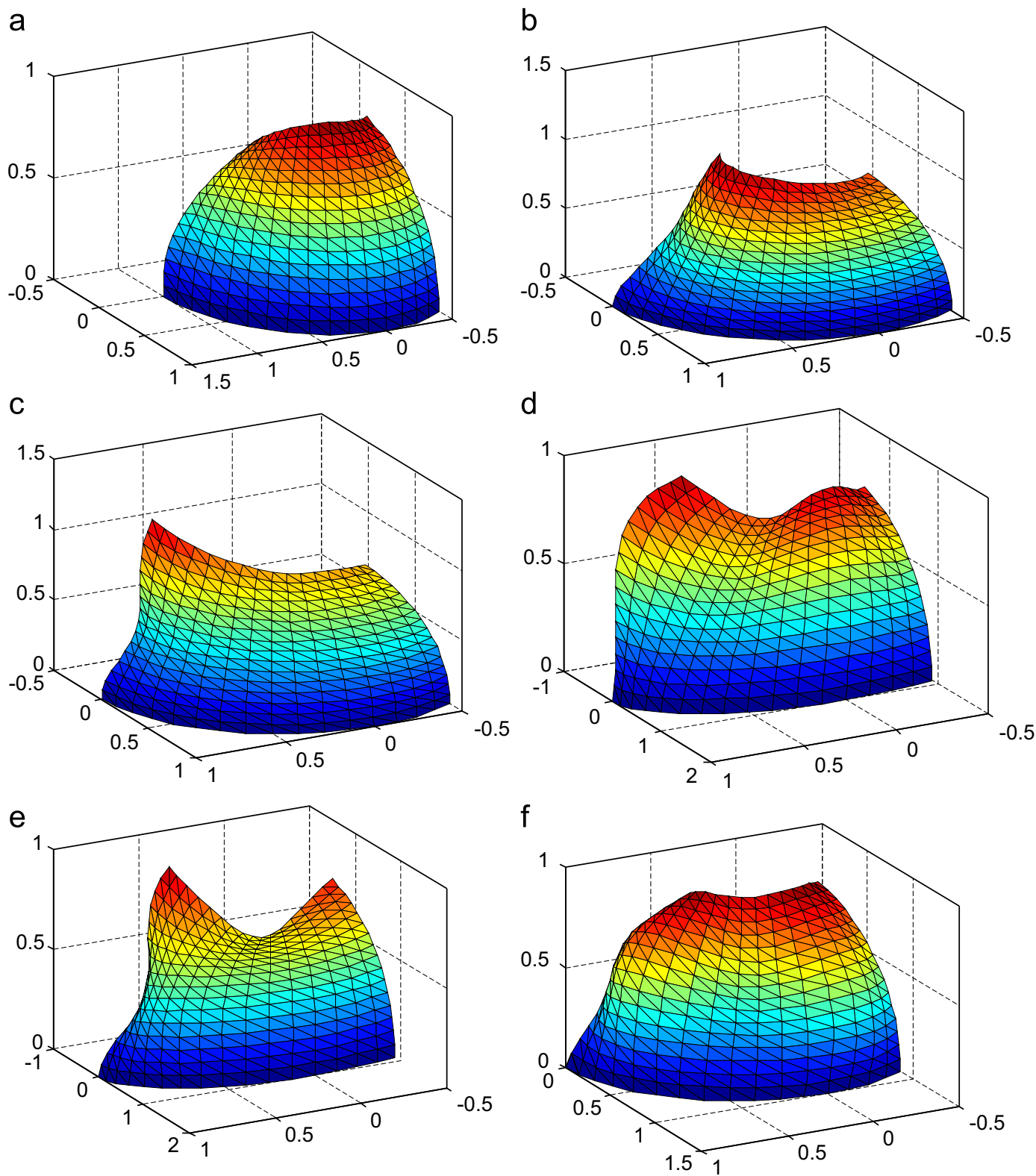

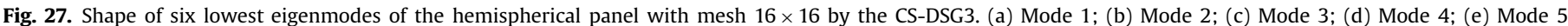
and (f) Mode 6. 


\section{Acknowledgments}

This work was supported by Vietnam National Foundation for Science \& Technology Development (NAFOSTED), Ministry of Science \& Technology, under the basic research program (Project no. 107.02-2012.05).

\section{References}

[1] Reddy JN. Theory and analysis of elastic plates and shells. NewYork: CRC Press: Taylor and Francis Group; 2006.

[2] Bathe KJ. Finite element procedures. New Jersey: Prentice-Hall; 1996.

[3] Zienkiewicz OC, Taylor RL. The finite element method, vol. 2. Solid mechanics. 5th ed.. Oxford: Butterworth-Heinemann; 2000.

[4] Henry Yang TY, Saigal S, Masud A, Kapania RK. A survey of recent shell finite elements. Int J Num Methods Eng 2000;47:101-27.

[5] Belytschko T, Leviathan I. Physical stabilization of the 4-node shell element with one-point quadrature. Comput Methods Appl Mech Eng 1994;113: 321-50.

[6] Dvorkin EN, Bathe KJ. A continuum mechanics based four-node shell element for general non-linear analysis. Eng Comput 1984;1:77-8.

[7] Flores FG, Estrada CF. A rotation-free thin shell quadrilateral. Comput Methods Appl Mech Eng 2007;196:2631-46.

[8] Zhang YX, Cheung YK, Chen WJ. Two refined non-conforming quadrilateral flat shell elements. Int J Num Methods Eng 2000;49:3355-82.

[9] Carpenter N, Stolarski H, Belytschko T. Improvements in 3-node triangular shell elements. Int J Num Methods Eng 1986;23:1643-7.

[10] Onate E, Zarate F, Flores F. A simple triangular element for thick and thin plate and shell analysis. Int J Num Methods Eng 1994;37:2569-82.

[11] Zienkiewicz OC, Taylor RL, Too JM. Reduced integration techniques in general of plates and shells. Int J Num Methods Eng 1971;3:275-90.

[12] Macneal RH. Derivation of element stiffness matrices by assumed strain distributions. Nucl Eng Des 1982;70:3-12.

[13] Bathe KJ, Dvorkin EN. A formulation of general shell elements-the use of mixed interpolation of tensorial components. In J Num Methods Eng 1986;22:697-722.

[14] Lee SL, Han SE. Free-vibration analysis of plates and shells with a nine-node assumed natural degenerated shell element. J Sound Vib 2001;241:605-33.

[15] Bletzinger KU, Bischoff M, Ramm E. A unified approach for shear-locking free triangular and rectangular shell finite elements. Comput Struct 2000;75: 321-34.

[16] Liu GR, Nguyen Thoi Trung. Smoothed finite element methods. New York: CRC Press, Taylor and Francis, Group; 2010.
[17] Liu GR, Dai KY, Nguyen-Thoi T. A smoothed finite element for mechanics problems. Comput Mech 2007;39:859-77.

[18] Liu GR, Nguyen-Thoi T, Dai KY, Lam KY. Theoretical aspects of the smoothed finite element method (SFEM). Int J Num Methods Eng 2007;71:902-30.

[19] Chen JS, Wu CT, Yoon S, You Y. A stabilized conforming nodal integration for galerkin mesh-free methods. Int J Num Methods Eng 2001;50:435-66.

[20] Nguyen-Thoi T, Phung-Van P, Luong-Van H, Nguyen-Van H, Nguyen-Xuan H. A cell-based smoothed three-node Mindlin plate element (CS-MIN3) for static and free vibration analyses of plates. Comput Mech 2013:50(1):65-81.

[21] Nguyen-Thoi T, Phung-Van P, Nguyen-Xuan H, Thai-Hoang C. A cell-based smoothed discrete shear gap method using triangular elements for static and free vibration analyses of Reissner-Mindlin plates. Int J Num Methods Eng 2012;91(7):705-41.

[22] Cui XY, Liu GR, Li GY, Zhao X, Nguyen-Thoi T, Sun GY. A smoothed finite element method (SFEM) for linear and geometrically nonlinear analysis of plates and shells. CMES-Comput Modeling Eng Sci 2008;28:2109-25.

[23] Nguyen-Thoi T, Phung-Van P, Rabczuk T, Nguyen-Xuan H, Le-Van C. An application of the ES-FEM in solid domain Q4 for dynamic analysis of 2D fluid-solid interaction problems. Int J Comput Methods 2013;10(1):1340003.

[24] Nguyen-Xuan H, Nguyen-Thoi T. A stabilized smoothed finite element method for free vibration analysis of Mindlin-Reissner plates. Commun Num Methods Eng 2009;25:882-906.

[25] Nguyen-Thoi T, Phung-Van P, Rabczuk T, Nguyen-Xuan H, Le-Van C. Free and forced vibration analysis using the n-sided polygonal cell-based smoothed finite element method (nCS-FEM). Int J Comput Methods 2013;10 (1):1340008.

[26] Bischoff M, Bletzinger KU. Stabilized DSG plate and shell elements.Trends in Computational structural mechanics. Barcelona, Spain: CIMNE; 2001.

[27] Lyly M, Stenberg R, Vihinen T. A stable bilinear element for the ReissnerMindlin plate model. Comput Methods Appl Mech Eng 1993;110:343-57.

[28] Batoz JL, Bathe KJ, Ho LW. A study of three-node triangular plate bending elements. Int J Num Methods Eng 1980;15:1771-812.

[29] Tessler A, Hughes TJR. A three-node mindlin plate element with improved transverse shear. ComputMethods Appl Mech Eng 1985;50:71-101.

[30] Bathe KJ, Dvorkin EN. A four-node plate bending element based on MindlinReissner plate theory and a mixed interpolation. Int J Num Methods Eng $1985 ; 21: 367-83$

[31] Fluge W. Stress in shells. Berlin: Springer; 1960

[32] Scordelis AC, Lo KS. Computer analysis of cylindrical shells. J Am Concr Inst 1964;61:539-61.

[33] McNeal RH, Harder RL. A proposed set of problems to test finite element accuracy. Finite Elem Anal 1985;1:3-20.

[34] Chapelle D, Bathe KJ, Iosilevich A. An evaluation of the MITC shell elements. Comput Struct 2000;75:1-30.

[35] Leissa AW. Vibration of shell. Washington DC: NASA, SP-288; 1973

[36] Francesco T, Erasmo V. Vibration analysis of spherical structural elements using the GDQ method. Comput Math Appl 2007;53:101538-60. 